\title{
ENDOGENOUS PARTICIPATION IN CHARITY AUCTIONS
}

\author{
by
}

Jeffrey Carpenter, Jessica Holmes and Peter Hans Matthews

September 2007

MIDDLEBURY COLLEGE ECONOMICS DISCUSSION PAPER NO. 07-07

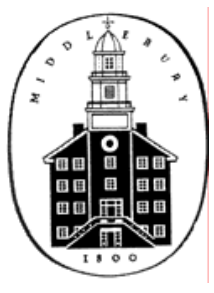

DEPARTMENT OF ECONOMICS

MIDDLEBURY COLLEGE

MIDDLEBURY, VERMONT 05753

http://www.middlebury.edu/ econ 


\title{
ENDOGENOUS PARTICIPATION IN CHARITY AUCTIONS*
}

\author{
Jeffrey Carpenter ${ }^{\dagger}$ Jessica Holmes ${ }^{\ddagger}$ Peter Hans Matthews ${ }^{\S}$
}

September 5, 2007

\begin{abstract}
Data from a recent field experiment suggests that differences in participation rates are responsible for much of the variations in revenues across formats in charity auctions. We provide a theoretical framework for the analysis of this, and other related, results. The model illustrates the limits of previous "fixed $N$ " results and introduces some new considerations to the choice of auction mechanism. It also implies, however, that the data cannot be explained in terms of participation costs alone: there must exist mechanism-specific obstacles to participation.
\end{abstract}

\section{Introduction}

From the small town silent auction that raises a few hundred dollars to the $\$ 70$ million Robin Hood annual benefit in New York City (Anderson 2007), charities and non-profits often use auctions to transform donations in kind into cash. The choice of format constitutes a difficult decision problem, however, even under idealized circumstances: if all bidders, win or lose, derive some benefit from monies raised, revenue equivalence does not hold, even if valuations of the object itself are private and independent. ${ }^{1} \quad$ Until recently, however, and despite an otherwise vibrant literature on the economics of auctions, little attention had been devoted to the properties, theoretical or otherwise, of charity auctions.

The best known result is perhaps Goeree et al's (2005) theorem, that when the standard (SIPV, or single object, independent private values) framework is extended so that all bidders receive some revenue proportional benefit, lotteries and winner-pay auctions produce less expected revenue than all-pay auctions. The intuition, as they characterize it, is that bids are suppressed under winner-pay mechanisms because when one bidder tops the others, she may win the object, but she also loses the chance to free ride on the benefits associated with the best of the other bids. While there are few,

\footnotetext{
${ }^{*}$ We thank Carolyn Craven, Steve Holmes and Corinna Noelke for comments, and the NSF (SES 0617778) for financial support.

${ }^{\dagger}$ Department of Economics, Middlebury College and IZA; jpc@middlebury.edu

${ }^{\ddagger}$ Department of Economics, Middlebury College; jholmes@middlebury.edu

$\S$ Department of Economics, Middlebury College; pmatthew@middlebury.edu

${ }^{1}$ This characteristic is not unique to charity auctions: Engelbrecht-Wiggans (1994) counts Amish estate sales and buyer ring knockouts as examples of auctions with what he calls "price proportional benefits."
} 
if any, examples of all-pay charity auctions, their result seems to rationalize the widespread use of raffles and lotteries, both inefficient variations on the all-pay theme. Engers and McManus (2006) have since shown that if bidders who contribute experience an additional "warm glow" (Andreoni 1995), the superiority of the all-pay over both first-price and second-price winner pay mechanisms survives in the limit, as the number of bidders increases.

Two recent lab experiments would seem to support this result. Davis et al (2006) find that lotteries raise more revenue than English auctions, while Goeree and Schram (2003) conclude that lotteries do worse than all-pay auctions but better than first price auctions. Our point of departure, however, is Carpenter, Holmes and Matthews (2006), one of the few field experiments on charity auctions, which reaches more or less the opposite conclusion, namely, that the all-pay mechanism produces no more revenue, in a statistical sense, than the second price sealed bid, and that both produce less revenue the first price sealed bid. As an empirical matter, the reversal owes much to differences in participation rates across mechanisms. While the model in Goeree et al (2005) and the designs in Goeree and Schram (2003) and Davis et al (2006) assume a fixed number of bidders - so that the separation of potential bidders into active and inactive bidders, a critical distinction in this paper, isn't relevant - the results in Carpenter, Holmes and Matthews (2006) indicate that the ratio of active to potential bidders, or the participation rate, is lower in second price than first price sealed bid auctions, and lower still in all-pay auctions.

The immediate purpose of this paper is to provide a theoretical framework for the analysis of these, and perhaps other, empirical observations on charity auctions. Such a framework should allow us to ask, for example, whether the existence of some sort of participation cost, and therefore an endogenous number of active bidders, is sufficient per se to reverse the ordering in Goeree et al (2005), or whether this requires mechanism-specific differences in costs. That is, is endogenous participation enough, on its own, to explain the underperformance of the all-pay auctions in Carpenter, Holmes and Matthews (2006), or should we conclude that the costs of participation in all-pay auctions are higher, either because bids are more difficult to calculate or bidders do not like the mechanism?

The next section derives the optimal symmetric bid and expected revenue functions for the first price, second price and all-pay sealed bid SIPV auctions in which all bidders, active or otherwise, earn a benefit that is proportional to revenue, and those who contribute to the charity (one bidder in winner-pay auctions, and all active bidders in all-pay auctions) experience a warm glow proportional to their bids, in an environment in which the submission of a bid imposes some cost on bidders. This representation of the participation problem owes much to the recent work of Menezes and Monteiro (2000) and, much earlier, Samuelson (1985). 
In the third section, we explore the properties of these bid and revenue functions, both within and across mechanisms, a more complicated task than first seems. From Menezes and Monteiro (2000), for example, we know that in the absence of revenue proportional benefits, the introduction of participation costs does not overturn revenue equivalence but can cause this still common revenue function to exhibit some unusual behavior: it need not be the case, for example, that expected revenue rises with the number of potential bidders, or that in the limit, it is independent of the distribution of private values. From Engers and McManus (2006), on the other hand, we learn that even without participation costs, there is no fixed order of revenues for small - that is, low $N$ - auctions. To cultivate a sense of what properties are, and are not, usual, we calculate and plot numerical bid and revenue functions for several members of the Kumaraswamy (1980) family of bounded value distributions.

\section{Optimal Bids and Expected Revenues}

\subsection{General Framework}

There are $N \geq 2$ potential risk neutral bidders whose private values for some indivisible object can be modeled as independent draws from some continuously differentiable distribution function $F$ over the unit interval $[0,1]$. These values are known to bidders before the decision to participate (or not) must be made. Auction revenues are used to provide a service from which all bidders, active or inactive, benefit: as in Goeree et al (2005), the value to each bidder is a constant fraction $0 \leq \alpha<1$ of these revenues. Some active bidders will also experience a "warm glow" (Andreoni 1995; Engers and McManus 2006) equal to a fraction $0 \leq \gamma<1-\alpha$ of their own contribution to auction revenue. The limit on $\gamma$, needed to ensure that each bidder's optimization problem is well-defined, implies that $\beta=\alpha+\gamma$, the sum of the common return and warm glow, is also less than one.

Following Samuelson (1985) and, more recently, Menezes and Monteiro (2000), potential bidders confront some cost of participation $0 \leq c^{j}<1, j=f$ (irst price), $s$ (econd price), a(ll pay), the value of which could be mechanism-specific. As a result, the number of active bidders is not predetermined. Samuelson (1985) and others define this cost in terms of the resources committed to "bid preparation," but on the basis of recent experimental evidence from both the lab and the field (Carpenter, Holmes and Matthews 2006, 2007), a broader definition is warranted, one that allows bidders to hold "intrinsic preferences" over mechanisms.

Within this framework, the derivation of optimal bid functions draws heavily on both Menezes and Monteiro (2000) and Engers and McManus (2006). In particular, symmetric Bayes-Nash 
equilibria are identified for bid functions $\sigma^{j}\left(v_{i}\right)$ that are assumed (for the moment, at least) to be differentiable above some participation threshold $0 \leq \underline{v}<1$.

\subsection{First Price Sealed Bid}

The representative bidder must decide whether or not to participate and, if she does, what type $\widehat{v}$ to announce or, equivalently, what bid $\sigma^{f}(\widehat{v})$ to submit. To this end, consider first the conditions under which someone with the private value $v \geq \underline{v}$ will find it optimal to reveal her true type when the participation threshold $\underline{v}$ is assumed fixed. With likelihood $C_{M}^{N-1} F(\underline{v})^{(N-1)-M}(1-F(\underline{v}))^{M}$, where $C_{q}^{p}=\frac{p !}{(p-q) ! q !}$, she will compete with $M$ other bidders for the object and, conditional on $M \geq 1$, the first order statistic of their values (that is, the maximum) has the distribution function $G(x, M)=(F(x)-F(\underline{v}))^{M} /(1-F(\underline{v}))^{M}$ If $M=0$, there will of course be no rivals and, therefore, no first order statistic. The conditional return on the $\operatorname{bid} \sigma^{f}(\widehat{v})$ for fixed $M \geq 1$ is then:

$$
E U(\widehat{v}, v, M)=\int_{\underline{v}}^{\widehat{v}}\left(v-(1-\beta) \sigma^{f}(\widehat{v})\right) g(x, M) d x+\alpha \int_{\widehat{v}}^{\bar{v}} \sigma^{f}(x) g(x, M) d x
$$

where $g(x, M)=d G(x, M) / d x=M(F(x)-F(\underline{v}))^{M-1} f(x) /(1-F(\underline{v}))^{M}$ is the conditional density function of the first order statistic. The first term in (1) represents the bidder's expected return when she wins the auction - because she earns both the common return $\alpha \sigma^{f}(\widehat{v})$ on her bid and experiences the warm glow $\gamma \sigma^{f}(\widehat{v})$ in this case, her "net bid" is $(1-(\alpha+\gamma)) \sigma^{f}(\widehat{v})=(1-\beta) \sigma^{f}(\widehat{v})$ while the second term is the expected benefit that still accrues to her when she loses.

It follows that the unconditional expected return, $E U(\widehat{v}, v)$, will be:

$$
\begin{aligned}
E U(\widehat{v}, v)= & F(\underline{v})^{N-1}\left(v-(1-\beta) \sigma^{f}(\widehat{v})\right) \\
& +\sum_{M=1}^{N-1} C_{M}^{N-1} F(\underline{v})^{N-1-M}(1-F(\underline{v}))^{M} E U(\widehat{v}, v, M) \\
= & F(\underline{v})^{N-1}\left(v-(1-\beta) \sigma^{f}(\widehat{v})\right) \\
& +\left(v-(1-\beta) \sigma^{f}(\widehat{v})\right) \sum_{M=1}^{N-1} C_{M}^{N-1} F(\underline{v})^{N-1-M}(1-F(\underline{v}))^{M} \int_{\underline{v}}^{\widehat{v}} g(x, M) d x \\
& +\alpha \sum_{M=1}^{N-1} C_{M}^{N-1} F(\underline{v})^{N-1-M}(1-F(\underline{v}))^{M} \int_{\widehat{v}}^{\bar{v}} B^{F}(x) g(x, M) d x \\
= & F(\underline{v})^{N-1}\left(v-(1-\beta) \sigma^{f}(\widehat{v})\right)+\left(F(\widehat{v})^{N-1}-F(\underline{v})^{N-1}\right)\left(v-(1-\beta) \sigma^{f}(\widehat{v})\right) \\
& +\alpha \sum_{M=1}^{N-1} C_{M}^{N-1} F(\underline{v})^{N-1-M} M \int_{\widehat{v}}^{1}(F(x)-F(\underline{v}))^{M-1} f(x) \sigma^{f}(x) d x
\end{aligned}
$$

after substitution for $G(x, M)$ and $g(x, M)$, where the first term on the right hand side of each equality is the expected return in the case where there are no other bidders, and the last equality follows from the fact that $\int_{\underline{v}}^{\widehat{v}} g(x, M)=(F(\widehat{v})-F(\underline{v}))^{M} /(1-F(\underline{v}))^{M}$ and that, as a consequence of the binomial theorem, $\sum_{M=1}^{N-1} C_{M}^{N-1} F(\underline{v})^{N-1-M}(F(\widehat{v})-F(\underline{v}))^{M}=F(\widehat{v})^{N-1}-F(\underline{v})^{N-1}$. 
The derivative of $E U(\widehat{v}, v)$ with respect to the bidder's choice variable $\widehat{v}$ is therefore:

$$
\begin{aligned}
\frac{\partial E U(\widehat{v}, v)}{\partial \widehat{v}}= & -(1-\beta) F(\widehat{v})^{N-1} \frac{d \sigma^{f}(\widehat{v})}{d \widehat{v}} \\
& +(N-1) F(\widehat{v})^{N-2} f(\widehat{v})\left(v-(1-\beta) \sigma^{f}(\widehat{v})\right) \\
& -\alpha f(\widehat{v}) \sigma^{f}(\widehat{v}) \sum_{M=1}^{N-1} C_{M}^{N-1} M F(\underline{v})^{N-1-M}(F(\widehat{v})-F(\underline{v}))^{M-1} \\
= & -(1-\beta) F(\widehat{v})^{N-1} \frac{d \sigma^{f}(\widehat{v})}{d \widehat{v}}+(N-1) F(\widehat{v})^{N-2} f(\widehat{v})\left(v-(1-\beta) \sigma^{f}(\widehat{v})\right) \\
& -\alpha(N-1) F(\widehat{v})^{N-2} f(\widehat{v}) \sigma^{f}(\widehat{v})
\end{aligned}
$$

where the second line follows from a corollary of the binomial theorem, $\sum_{M=1}^{N-1} C_{M}^{N-1} M F(\underline{v})^{N-1-M}(F(\widehat{v})-$ $F(\underline{v}))^{M-1}=(N-1) F(\underline{v})^{N-2}$. The first order condition for a SBNE is that $\frac{\partial E U(\widehat{v}, v)}{\partial \widehat{v}}=0$ at $\widehat{v}=v$, which leads, after some simplification, to the first order differential equation:

$$
\frac{d \sigma^{f}(v)}{d v}+\frac{(N-1)(1-\gamma)}{(1-\beta)} \frac{f(v)}{F(v)} \sigma^{f}(v)=\frac{(N-1)}{(1-\beta)} \frac{f(v)}{F(v)} v
$$

While (4) is not exact, there exists an integrating factor, $F(v)^{\theta}$, where $\theta=\frac{(N-1)(1-\gamma)}{(1-\beta)}$, so that:

$$
\frac{d\left(\sigma^{f}(v) F(v)^{\theta}\right)}{d v}=\frac{N-1}{1-\beta} F(v)^{\theta-1} f(v) v
$$

or:

$$
\sigma^{f}(v) F(v)^{\theta}=\frac{N-1}{1-\beta} \int F(x)^{\theta-1} f(x) x d x+k
$$

where $k$ is a constant of integration. Because the optimal threshold bid, $\sigma^{f}(\underline{v})$, and therefore the product $\sigma^{f}(\underline{v}) F(\underline{v})^{\theta}$, are both zero, it follows that ${ }^{2}$ :

$$
\sigma^{f}(v)=\frac{N-1}{(1-\beta)} \frac{1}{F(v)^{\theta}} \int_{\underline{v}}^{v} F(x)^{\theta-1} f(x) x d x
$$

or, after integration by parts and further simplification:

$$
\sigma^{f}(v)=\frac{1}{1-\gamma}\left[v-\frac{F(\underline{v})^{\theta}}{F(v)^{\theta}} \underline{v}-\frac{1}{F(v)^{\theta}} \int_{\underline{v}}^{v} F(x)^{\theta} d x\right]
$$

Inasmuch as the participation threshold is not predetermined, however, the optimal bid function (8) is not a reduced form. To this end, recall that the revenue proportional benefits of the auction are not conditional on participation, and observe that a potential bidder with private value $\underline{v}$ should be indifferent between participation (and the submission of a zero bid) and non-participation. If such a bidder does participate, the likelihood that she will win the auction is $F(\underline{v})^{N-1}$, in which case she

\footnotetext{
${ }^{2}$ In Engers and McManus (2006), the optimal bid at the "threshold" - in their case, the lower limit on the compact support of $F$ - is indeterminate. The difference is that, in their case, the likelihood that a bidder with the threshold value wins the auction is zero.
} 
receives a benefit equal to her private value $\underline{v}$. (Since $\sigma^{f}(\underline{v})=0$, there is neither a common return nor a warm glow.) With likelihood $C_{M}^{N-1} F(\underline{v})^{(N-1)-M}(1-F(\underline{v}))^{M}$, on the other hand, she will lose the auction to one of $M \geq 1$ other bidders, but receive a benefit that is equal to a fraction $\alpha$ of the expected maximum bid, or $\alpha \int_{\underline{v}}^{1} g(x, M) \sigma^{f}(x) d x$. The net benefit of participation is therefore:

$$
F(\underline{v})^{N-1} v+\alpha \sum_{M=1}^{N-1} C_{M}^{N-1} F(\underline{v})^{(N-1)-M}(1-F(\underline{v}))^{M} \int_{\underline{v}}^{1} g(x, M) \sigma^{f}(x) d x-c^{f}
$$

where $c^{f}$ is the cost of participation in a (f)irst price auction. The net benefit of non-participation is equal to:

$$
\alpha \sum_{M=1}^{N-1} C_{M}^{N-1} F(\underline{v})^{(N-1)-M}(1-F(\underline{v}))^{M} \int_{\underline{v}}^{1} g(x, M) \sigma^{f}(x) d x
$$

since the externalities that other bidders produce are not limited to participants. The "threshold bidder" is therefore someone for whom:

$$
F(\underline{v})^{N-1} \underline{v}=c^{f}
$$

This condition defines an implicit function in which the participation threshold $\underline{v}$ depends on the costs of participation $c^{f}$, the number of potential bidders $N$ and, implicitly, the shape of the distribution function $F(v)$. If the effects of the first are more or less predictable - if potential bidders have better outside options, fewer of them will participate - the implications of the second are more subtle and call for some comment. As the number of potential bidders increases, so, too, does the likelihood that a particular active bidder will lose whatever she has "invested" in the auction which, in turn, causes the threshold to rise. It is then not obvious that an increase in the number of potential bidders or, if one prefers, auction size, will always lead to an increase in the expected number of active bidders and, so, expected revenue.

It is important to note, however, that this participation effect is not the result of some increased desire to free ride on the contributions of other bidders. The threshold $\underline{v}$ in (11) does not depend on either the common return $\alpha$ or warm glow $\gamma$ : it is the same condition, in fact, that Menezes and Monteiro (2002) derive for their "no spillover" model. The reason is that non-participants benefit from these spillovers, too.

Charities will be less interested in bid functions and their properties than expected revenue $R^{f}$ and, to this end, we note that since the density function of the first order statistic for all $N$ private values is $N F(v)^{N-1} f(v), R^{f}$ will be equal to: 


$$
R^{f}=N \int_{\underline{v^{f}}\left(c^{f}, N\right)}^{1} F(v)^{N-1} f(v) \sigma^{f}(v) d v
$$

where the threshold value is written $\underline{v^{f}}\left(c^{f}, N\right)$ as a reminder that the lower limit is not fixed in the usual sense.

\subsection{Second Price Sealed Bid Auction}

The derivation of the SBNE bid and expected revenue functions in the second price auction calls for the introduction of another distribution function, $J(x, M)$, the conditional distribution of the second order statistic for private values when there are $M \geq 2$ other active bidders:

$$
J(x, M)=M\left(\frac{F(x)-F(\underline{v})}{1-F(\underline{v})}\right)^{M-1}-(M-1)\left(\frac{F(x)-F(\underline{v})}{1-F(\underline{v})}\right)^{M}
$$

It will also be useful to note that the likelihood that a bidder who announces type $\widehat{v}$ is the runner-up is:

$$
M\left(\frac{F(\widehat{v})-F(\underline{v})}{1-F(\underline{v})}\right)^{M-1}\left(1-\frac{F(\widehat{v})-F(\underline{v})}{1-F(\underline{v})}\right)=\frac{M(F(\widehat{v})-F(\underline{v}))^{M-1}(1-F(\widehat{v}))}{(1-F(\underline{v}))^{M}}
$$

since it is her bid, $\sigma^{s}(\widehat{v})$, that determines the winner's payment.

With this in mind, with likelihood $F(\underline{v})^{N-1}$, where $\underline{v}$ once more denotes the relevant participation threshold, the representative bidder will have no active competitors. If it is assumed that in an auction with one bidder, the "second price" is zero, then such a bidder would earn a benefit of $v$, no matter what bid $\sigma^{s}(\widehat{v})$ she submits.

With likelihood $(N-1) F(\underline{v})^{N-2}(1-F(\underline{v}))$, on the other hand, she will compete with just one other bidder $(M=1)$, with expected benefits equal to:

$$
\begin{aligned}
E U(\widehat{v}, v, 1) & =\int_{\underline{v}}^{\widehat{v}}\left(v-(1-\beta) \sigma^{s}(x)\right) g(x, 1) d x+\frac{(1-F(\widehat{v}))}{(1-F(\underline{v}))} \alpha \sigma^{s}(\widehat{v}) \\
& =\frac{1}{(1-F(\underline{v}))} \int_{\underline{v}}^{\widehat{v}}\left(v-(1-\beta) \sigma^{s}(x)\right) f(x) d x+\frac{(1-F(\widehat{v}))}{(1-F(\underline{v}))} \alpha \sigma^{s}(\widehat{v})
\end{aligned}
$$

The first term is the (conditional on $M=1$ ) expected benefit when she wins - the difference between this term and its equivalent under the first price mechanism is that the relevant bid is now $\sigma^{s}(x)$ rather than $\sigma^{s}(\widehat{v})$ - and the second captures the fact that when she loses, the value of her bid, $\sigma^{s}(\widehat{v})$, determines the winner's payment and therefore the value of the common benefit.

Finally, she will face $M \geq 2$ competitors with likelihood $C_{M}^{N-1} F(\underline{v})^{N-1-M}(1-F(\underline{v}))^{M}$, with 
expected benefits:

$$
\begin{aligned}
E U(\widehat{v}, v, M)= & \int_{\underline{v}}^{\widehat{v}}\left(v-(1-\beta) \sigma^{s}(x)\right) g(x, M) d x \\
& +\frac{M(F(\widehat{v})-F(\underline{v}))^{M-1}(1-F(\widehat{v}))}{(1-F(\underline{v}))^{M}} \alpha \sigma^{s}(\widehat{v}) \\
& +\alpha \int_{\widehat{v}}^{1} \sigma^{s}(x) j(x, M) d x
\end{aligned}
$$

where:

$$
j(x, M)=\frac{d J(x, M)}{d x}=\frac{M(M-1)(F(x)-F(\underline{v}))^{M-2}(1-F(x)) f(x)}{(1-F(\underline{v}))^{M}}
$$

is the density function of the second order statistic. As before, the first and second terms represent, respectively, the expected benefits when she wins, and when she loses but submits the second highest bid. The additional third term measures the direct spillover when she is neither the first nor second price bidder.

With some simplification, the unconditional return $E U(v, \widehat{v})$ can then be written:

$$
\begin{aligned}
E U(v, \widehat{v})= & F(\underline{v})^{N-1} v+(N-1) F(\underline{v})^{N-2} \int_{\underline{v}}^{\widehat{v}}\left(v-(1-\beta) \sigma^{s}(x)\right) f(x) d x \\
& +\alpha(N-1) F(\underline{v})^{N-2}(1-F(\widehat{v})) \sigma^{s}(\widehat{v}) \\
& +\sum_{M=2}^{N-1} C_{M}^{N-1} F(\underline{v})^{N-1-M} M\left(\int_{\underline{v}}^{\widehat{v}}\left(v-(1-\beta) \sigma^{s}(x)\right)\left(F(x)-F(\underline{v})^{M-1} f(x) d x\right)\right. \\
& +\alpha(1-F(\widehat{v})) \sigma^{s}(\widehat{v}) \sum_{M=2}^{N-1} C_{M}^{N-1} M F(\underline{v})^{N-1-M}(F(\widehat{v})-F(\underline{v}))^{M-1} \\
& +\alpha \sum_{M=2}^{N-1} C_{M}^{N-1} M(M-1) F(\underline{v})^{N-1-M}\left(\int_{\widehat{v}}^{1} \sigma^{s}(x)(F(x)-F(\underline{v}))^{M-2}(1-F(x)) f(x) d x\right)
\end{aligned}
$$

The effects of variation in $\widehat{v}$ on $E U(v, \widehat{v})$ are a little easier to calculate than first seem because the derivatives of the fifth and sixth terms each contain, with opposite signs, the term $\alpha(1-F(\widehat{v})) \sigma^{s}(\widehat{v}) \sum_{M=2}^{N-1} C_{M}^{N-1} M(M-1) F(\underline{v})^{N-1-M}(F(\widehat{v})-F(\underline{v}))^{M-2} f(\widehat{v})$. It follows that:

$$
\begin{aligned}
\frac{\partial E U(v, \widehat{v})}{\partial \widehat{v}}= & (N-1) F(\underline{v})^{N-2}\left(v-(1-\beta) \sigma^{s}(\widehat{v})\right) f(\widehat{v}) \\
& +\alpha(N-1) F(\underline{v})^{N-2}\left[(1-F(\widehat{v})) \frac{d \sigma^{s}(\widehat{v})}{d \widehat{v}}-\sigma^{s}(\widehat{v}) f(\widehat{v})\right] \\
& +\left(v-(1-\alpha) \sigma^{s}(\widehat{v})\right) f(\widehat{v}) \sum_{M=2}^{N-1} M F(\underline{v})^{N-1-M}(F(\widehat{v})-F(\underline{v}))^{M-1} \\
& +\alpha\left[(1-F(\widehat{v})) \frac{d \sigma^{s}(\widehat{v})}{d \widehat{v}}-f(\widehat{v}) \sigma^{s}(\widehat{v})\right] \sum_{M=2}^{N-1} M F(\underline{v})^{N-1-M}(F(\widehat{v})-F(\underline{v}))^{M-1}
\end{aligned}
$$

The observation that, as a further consequence of the binomial theorem, $\sum_{M=2}^{N-1} M F(\underline{v})^{N-1-M}(F(\widehat{v})-$ $F(\underline{v}))^{M-1}=(N-1)\left(F(\widehat{v})^{N-2}-F(\underline{v})^{N-2}\right)$, and the requirement that $\partial E U(v, \widehat{v}) / \partial \widehat{v}=0$ at $v=\widehat{v}$ 
in equilibrium leads, after considerable simplification, to:

$$
\left(v-(1-\beta) \sigma^{s}(v)\right) f(v)+\alpha\left[(1-F(v)) \frac{d \sigma^{s}(v)}{d v}-f(v) \sigma^{s}(v)\right]=0
$$

or, if $v \neq 1$ and $\alpha \neq 0$, to another first order differential equation ${ }^{3}$ :

$$
\frac{d \sigma^{s}(v)}{d v}-\frac{(1-\gamma)}{\alpha} \frac{f(v)}{(1-F(v))} \sigma^{s}(v)=-\frac{1}{\alpha} \frac{f(v)}{(1-F(v))} v
$$

Multiplication of both sides of $(21)$ by the integrating factor $(1-F(v))^{\frac{1-\gamma}{\alpha}}$ then produces:

$$
\frac{d\left((1-F(v))^{\frac{1-\gamma}{\alpha}} \sigma^{s}(v)\right)}{d v}=-\frac{1}{\alpha} v f(v)(1-F(v))^{\frac{1-\beta}{\alpha}}
$$

or:

$$
(1-F(v))^{\frac{1-\gamma}{\alpha}} \sigma^{s}(v)=-\frac{1}{\alpha} \int v f(v)(1-F(v))^{\frac{1-\beta}{\alpha}}+k
$$

where $k$ is the constant of integration.

The choice of boundary condition, and therefore the calculation of $k$, is complicated for two reasons. The optimal threshold bid $\sigma^{s}(\underline{v})$ is, for reasons noted earlier, indeterminate, but the derivation of $(23)$ assumed that $v \neq 1$. The second problem can be circumvented if the domain of $(1-F(v))^{\frac{1-\gamma}{\alpha}} \sigma^{s}(v)$ is (re)extended such that $(1-F(1))^{\frac{1-\gamma}{\alpha}} \sigma^{s}(1)$ assumes its limit value of 0 . It then follows that:

$$
(1-F(v))^{\frac{1-\gamma}{\alpha}} \sigma^{s}(v)=\frac{1}{\alpha} \int_{v}^{1} x f(x)(1-F(x))^{\frac{1-\beta}{\alpha}} d x
$$

Integration by parts then implies:

$$
(1-F(v))^{\frac{1-\gamma}{\alpha}} \sigma^{s}(v)=\frac{1}{1-\gamma}(1-F(v))^{\frac{1-\gamma}{\alpha}} v+\frac{1}{1-\gamma} \int_{v}^{1}(1-F(x))^{\frac{1-\gamma}{\alpha}} d x
$$

or, if one assumes, once more, that $v \neq 1$, so that both sides can be divided by $(1-F(v))^{\frac{1-\gamma}{\alpha}}$ :

$$
\sigma^{s}(v)=\frac{1}{1-\gamma} v+\frac{1}{(1-\gamma)(1-F(v))^{\frac{1-\gamma}{\alpha}}} \int_{v}^{1}(1-F(x))^{\frac{1-\gamma}{\alpha}} d x
$$

The limit bids $\sigma^{s}(\underline{v})$ and $\sigma^{s}(1)$ are then chosen so that $\sigma^{s}(v)$ is continuous over the entire interval $[\underline{v}, 1]$

It isn't difficult to infer from (26) that, conditional on participation, neither the introduction of spillover effects nor participation costs causes bidders to become " $N$ sensitive." This should not come as much of a surprise, however, because Menezes and Monteiro (2002) show that it is

\footnotetext{
${ }^{3}$ If there is no common return - that is, if $\alpha=0$ - then (20) collapses to $\sigma^{s}(v)=(1-\gamma)^{-1} v$, a variation on the standard proposition that in a second price auction with independent private values, individuals will bid these values. In this case, individuals bid $\gamma(1-\gamma)^{-1}$ percent more than their values because it is possible, at least in principle, that there remains a warm glow $\gamma$.
} 
(still) dominant to bid one's value in the absence of the former, while Engers and McManus (2006) determine that in a second price charity auction with a fixed number of bidders, the optimal bid is independent of $N$.

Menezes and Monteiro (2002) also found, however, that the participation thresholds for first and second price auctions were equal, a result that is not robust with respect to the presence of a common return. To understand the difference, consider, once more, the situation faced by the "threshold bidder." If she participates, then with likelihood $F(\underline{v})^{N-1}$ she alone will submit a bid, and therefore win the object worth $\underline{v}$ to her at a cost of 0 , since there is no second price. With likelihood $(N-1) F(\underline{v})^{N-2}(1-F(\underline{v}))$, on the other hand, there will be a second bidder, someone who will (almost certainly) win at a cost of $\sigma^{s}(\underline{v})$, which produces a benefit of $\alpha \sigma^{s}(\underline{v})$ to the threshold bidder. Last, with likelihood $C_{M}^{N-1} F(\underline{v})^{N-M-1}(1-F(\underline{v}))^{M}$, there will be $M \geq 2$ other active bidders, and with no chance that the threshold bidder will determine the second price, the expected benefits that will accrue to her are $\alpha \sum_{M=2}^{N-1} C_{M}^{N-1} F(\underline{v})^{N-M-1}(1-F(\underline{v}))^{M} \int_{\underline{v}}^{1} j(x, M) \sigma^{s}(x) d x$, where, as defined earlier, $j(x, M)$ is the conditional density of the second order statistic. The net benefits of participation are therefore:

$$
\begin{aligned}
& F(\underline{v})^{N-1} \underline{v}+\alpha(N-1) F(\underline{v})^{N-2}(1-F(\underline{v})) \sigma^{s}(\underline{v}) \\
& +\alpha \sum_{M=2}^{N-1} C_{M}^{N-1} F(\underline{v})^{N-M-1}(1-F(\underline{v}))^{M} \int_{\underline{v}}^{1} j(x, M) \sigma^{s}(x) d x-c^{s}
\end{aligned}
$$

If, on the other hand, the threshold bidder does not participate, she receives 0 with likelihood $F(\underline{v})^{N-1}+(N-1) F(\underline{v})^{N-2}(1-F(\underline{v}))$, the likelihood that one or fewer bids are submitted, since there are no revenue proportional benefits in this case, and $\alpha \int_{\underline{v}}^{1} j(x, M) \sigma^{s}(x) d x$ with likelihood $C_{M}^{N-1} F(\underline{v})^{N-M-1}(1-F(\underline{v}))^{M}$ for $M \geq 2$. The net benefits of non-participation are therefore:

$$
\alpha \sum_{M=2}^{N-1} C_{M}^{N-1} F(\underline{v})^{N-M-1}(1-F(\underline{v}))^{M} \int_{\underline{v}}^{1} j(x, M) \sigma^{s}(x) d x
$$

The condition that defines the threshold $\underline{v}$ is therefore:

$$
F(\underline{v})^{N-1} \underline{v}+\alpha(N-1) F(\underline{v})^{N-2}(1-F(\underline{v})) \sigma^{s}(\underline{v})=c^{s}
$$

the solution of which will be denoted $\underline{v}^{s}=\underline{v}^{s}\left(N, c^{s}, \alpha\right)$. Relative to the first price threshold (11), two related properties of $\underline{v}^{s}\left(N, c^{s}, \alpha\right)$ call for attention. First, the threshold is now sensitive to the common return $\alpha$ and warm glow $\gamma=\beta-\alpha$ associated with the charity. Second, when participation costs are the same, $c^{f}=c^{s}$, the threshold is lower or, if one prefers, participation rates are higher, in the second price auction. A comparison of the two conditions (11) and (28) reveals that the 
difference is the term $\alpha(N-1) F(\underline{v})^{N-2}(1-F(\underline{v})) \sigma^{s}(\underline{v})$, the benefit that accrues to a threshold bidder in second price auction when there is just one other bidder, and she determines the winner's payment.

Expected revenues in the second price auction $R^{s}$ are therefore:

$$
\begin{aligned}
R^{s}= & N(N-1) \int_{\underline{\underline{v}}^{s}\left(N, c^{s}, \alpha, \beta\right)}^{1} F(x)^{N-2}(1-F(x)) f(x) \sigma^{s}(x) d x \\
= & \frac{N(N-1)}{1-\gamma}\left(\int_{\underline{v}^{s}\left(N, c^{s}, \alpha, \beta\right)}^{1} F(x)^{N-2}(1-F(x)) f(x) x d x\right. \\
& \left.\left.+\int_{\underline{v}^{s}\left(N, c^{s}, \alpha, \beta\right)}^{1} F(x)^{N-2}(1-F(x)) \int_{x}^{1}(1-F(z))^{\frac{1-\gamma}{\alpha}} d z d x\right)\right)
\end{aligned}
$$

where $N(N-1) F(v)^{N-2}(1-F(v)) f(v)$ is the unconditional density function of the second order statistic and the second line follows from substitution for $\sigma^{S}(x)$.

\subsection{All-Pay Sealed Bid Auction}

The derivation of the SBNE bid functions under the all-pay mechanism follows now familiar lines. With likelihood $F(\underline{v})^{N-1}$, the representative bidder will have no active rivals, and can expect $(v-$ $\left.(1-\beta) \sigma^{a}(\widehat{v})\right)$. With likelihood $C_{M}^{N-1} F(\underline{v})^{N-1-M}(1-F(\underline{v}))^{M}$, she will have $M \geq 1$ rivals, and expect:

$$
\begin{aligned}
E U(\hat{v}, v, M) & =\int_{\underline{v}}^{\widehat{v}} v g(x, M) d x+\frac{\alpha M}{(1-F(\underline{v}))} \int_{\underline{v}}^{1} f(x) \sigma^{a}(x) d x-(1-\beta) \sigma^{a}(\widehat{v}) \\
& =\frac{(F(\widehat{v})-F(\underline{v}))^{M}}{(1-F(\underline{v}))^{M}} v+\frac{\alpha M}{(1-F(\underline{v}))} \int_{\underline{v}}^{1} f(x) \sigma^{a}(x) d x-(1-\beta) \sigma^{a}(\widehat{v})
\end{aligned}
$$

The first term reflects the fact that she will win the auction, and receive her private value $v$, with likelihood $G(\widehat{v}, M)$. The second and third follow from the observation that, win or lose, she will forfeit the net cost of her bid, $(1-\beta) \sigma^{a}(\widehat{v})$, but obtain benefits equal to a fraction $\alpha$ of the sum of all other bids, expressed here as the product of the number of active bidders $M$ and the mean bid $\int_{\underline{v}}^{1} \frac{f(x)}{(1-F(\underline{v}))} \sigma^{a}(x) d x$. Substitution for $g(x, M)$ in the first term and integration then leads to the second line.

After some simplification, the unconditional payoff $E U(v, \hat{v})$ for a bidder who assumes type $\hat{v}$ is therefore:

$$
\begin{aligned}
E U(v, \hat{v})= & F(\underline{v})^{N-1}\left(v-(1-\beta) \sigma^{a}(\widehat{v})\right) \\
& +v \sum_{M=1}^{N-1} C_{M}^{N-1} F(\underline{v})^{N-1-M}(F(\widehat{v})-F(\underline{v}))^{M} \\
& +\frac{\alpha}{(1-F(\underline{v}))} \int_{\underline{v}}^{1} \sigma^{a}(x) f(x) d x \sum_{M=1}^{N-1} C_{M}^{N-1} M F(\underline{v})^{N-1-M}(1-F(\underline{v}))^{M} \\
& -(1-\beta) \sigma^{a}(\widehat{v}) \sum_{M=1}^{N-1} C_{M}^{N-1} F(\underline{v})^{N-1-M}(1-F(\underline{v}))^{M}
\end{aligned}
$$


or recalling that $\sum_{M=1}^{N-1} C_{M}^{N-1} F(\underline{v})^{N-1-M}(1-F(\underline{v}))^{M}=1-F(\underline{v})^{N-1}$ and $\sum_{M=1}^{N-1} C_{M}^{N-1} M F(\underline{v})^{N-1-M}(1-$ $F(\underline{v}))^{M}=(N-1)(1-F(\underline{v}))$, and then noting that $\sum_{M=1}^{N-1} C_{M}^{N-1} F(\underline{v})^{N-1-M}(F(\widehat{v})-F(\underline{v}))^{M}=$ $F(\widehat{v})^{N-1}-F(\underline{v})^{N-1}$ :

$$
\begin{aligned}
E U(v, \hat{v})= & F(\underline{v})^{N-1}\left(v-(1-\beta) \sigma^{a}(\widehat{v})\right)+v\left(F(\widehat{v})^{N-1}-F(\underline{v})^{N-1}\right) \\
& +\alpha(N-1) \int_{\underline{v}}^{1} \sigma^{a}(x) f(x) d x-(1-\beta)\left(1-F(\underline{v})^{N-1}\right) \sigma^{a}(\widehat{v}) \\
= & F(\widehat{v})^{N-1} v+\alpha(N-1) \int_{\underline{v}}^{1} \sigma^{a}(x) f(x) d x-(1-\beta) \sigma^{a}(\widehat{v})
\end{aligned}
$$

The derivative of $E U(v, \hat{v})$ with respect to $\hat{v}$ is therefore just $v(N-1) F(\widehat{v})^{N-2} f(\widehat{v})-(1-\beta) \frac{d \sigma^{a}(\widehat{v})}{d \hat{v}}$, which equals zero at $\hat{v}=v$ if:

$$
\frac{d \sigma^{a}(v)}{d v}=\frac{N-1}{1-\beta} F(v)^{N-2} f(v) v
$$

The solution to this differential equation:

$$
\sigma^{a}(v)=\frac{N-1}{1-\beta} \int F(v)^{N-2} f(v) v+k
$$

where $k$ is a constant of integration. Since it is optimal for bidders with threshold values to bid zero, $\sigma^{a}(\underline{v})=0$, this becomes:

$$
\sigma^{a}(v)=\frac{N-1}{1-\beta} \int_{\underline{v}}^{v} F(x)^{N-2} f(x) x d x
$$

or, after integration by parts:

$$
\sigma^{a}(v)=\frac{1}{1-\beta}\left(v F(v)^{N-1}-\underline{v} F(\underline{v})^{N-1}\right)-\frac{1}{1-\beta} \int_{\underline{v}}^{v} F(x)^{N-1} d x
$$

If the costs of participation in first price and all-pay auctions are the same, then so, too, are the participation thresholds. ${ }^{4} \quad$ To show this, recall that with likelihood $F(\underline{v})^{N-1}$, the threshold bidder will be the lone participant, and win a prize worth $\underline{v}$ to her for a bid of 0 . With likelihood $C_{M}^{N-1} F(\underline{v})^{N-1-M}(1-F(\underline{v}))^{M}$, there will be $M \geq 1$ other bidders, each of whom will submit, in expectation, a bid equal to $\int_{\underline{v}}^{1} \sigma^{a}(x) f(x) d x$, which produces a benefit equal to $\alpha M \int_{\underline{v}}^{1} \sigma^{a}(x) f(x) d x$ for the threshold bidder. The net benefits of participation are therefore:

$$
F(\underline{v})^{N-1} \underline{v}+\alpha \sum_{M=1}^{N-1} C_{M}^{N-1} F(\underline{v})^{N-1-M}(1-F(\underline{v}))^{M} M \int_{\underline{v}}^{1} \sigma^{a}(x) f(x) d x-c^{a}
$$

The net benefits of non-participation, on the other hand, are:

\footnotetext{
${ }^{4}$ If costs are the same across all three mechanisms, then, participation will be lower in both the first price and all-pay than the second price.
} 


$$
\alpha \sum_{M=1}^{N-1} C_{M}^{N-1} F(\underline{v})^{N-1-M}(1-F(\underline{v}))^{M} M \int_{\underline{v}}^{1} \sigma^{a}(x) f(x) d x
$$

since, with likelihood $C_{M}^{N-1} F(\underline{v})^{N-1-M}(1-F(\underline{v}))^{M}$, there will be $M \geq 1$ other bidders who produce the same non-exclusive benefit of $\alpha M \int_{\underline{v}}^{1} \sigma^{a}(x) f(x) d x$. The threshold is therefore defined by:

$$
F(\underline{v})^{N-1} \underline{v}=c^{a}
$$

the solution of which is denoted $\underline{v}^{a}=\underline{v}^{a}\left(N, c^{a}\right)$.

The same demonstration also shows that expected revenues under the all-pay mechanism are equal to:

$$
R^{a}=N \int_{\underline{v}^{a}\left(N, c^{a}\right)}^{1} \sigma^{a}(v) f(v) d v
$$

\section{Comparison of Mechanisms}

\subsection{Numerical Analysis and the Kumaraswamy Distribution}

We observed, in the introduction, that there is no fixed order for the three mechanisms. This does not mean, however, that the mechanisms are without "common properties" that should inform both research and practice. To determine whether such properties exist, we shall compare numerical participation, bid and revenue functions when the distribution of private values over the unit interval is a member of the Kumaraswamy (1980) family:

$$
F(v \mid a, b)=1-\left(1-x^{a}\right)^{b} \quad a, b>0
$$

with mean $b \Gamma\left(1+\frac{1}{a}\right) \Gamma(b) / \Gamma\left(1+\frac{1}{a}+b\right)$. Much of the discussion that follows will focus on the four particular examples with the implied density functions depicted in Figure 1: $F(v \mid 1,1)$, the standard uniform distribution with mean 0.50 , and a benchmark in the literature; $F(v \mid 2,2)$, which has almost the same mean as the uniform distribution (0.53) but is hump-shaped, the equivalent of an auction in which few "extreme bidders" should be expected; $F(v \mid 3,1)$, with mean 0.25 , which produces auctions with an expected preponderance of "low value bidders"; and $F(v \mid 1,5)$, with mean 0.83 , which instead leads to auctions with a disproportionate number of "high value bidders."

\subsection{Threshold Values and Participation Rates}

We have shown that the threshold values under the first price and all-pay mechanisms are equal, and Table 1 reports the values of this common threshold and the implied non-participation rates 
as the auction size or number of potential bidders $N$ and participation cost $c$ vary for each of the four distributions of private values. One of the most immediate implications of this data is how much even small participation costs influence behavior. When the distribution of private values is bell-shaped, for example, the difference between $c=0$, or costless participation, and $c=0.01$, in which costs are just $\frac{1}{50}$ th of the mean private value, or $\frac{1}{100}$ th of the maximum private value, is the difference between no threshold and one that is equal to 0.46 in the $N=5$ case. From another perspective, there is now an almost 1 percent chance $\left(0.0079=(0.38)^{5}\right)$ chance that no one will submit a bid, despite the fact that there are few low value bidders. In practical terms, small obstacles to participation under either mechanism can drive numerous (potential) bidders from the auction.

Furthermore, in small auctions, even a small increase in the number of potential bidders induces a substantial increase in the threshold. In the uniform case when $c=0.01$, for example, the threshold rises from 0.10 to 0.40 as $N$ increases from 2 to 5 , and when $N=20$, which, for most purposes, is still a small auction, the threshold rises to 0.79. To provide a more intuitive characterization of the same phenomenon, increases in the number of potential bidders produce small, and ever smaller, increases in the expected number of active bidders, from $3=5(0.6)$ when $N=5$ to $3.7=10(0.37)$ when $N=10$, and then to $4.2=20(0.21)$ when $N=20$. In this particular case, in other words, the addition of 15 more potential bidders caused the expected number of active bidders to increase by little more than 1 .

There are at least two senses in which the pattern is a robust one. First, while it is possible to construct examples in which, over some short interval, the expected number of active bidders falls as the number of potential bidders rises, in none of the cases represented in Table 1, or for that matter Table 2, does this happen. Second, and to our initial surprise, for a fixed participation $\operatorname{cost} c$, the relationship between auction size and the number of active bidders doesn't vary much with the distribution of private values. Consider, for example, the situation in which $c=0.05$ and $N=10$. While the threshold value varies from 0.70 in the auction with few extreme bidders to, on the one hand, 0.41 in the auction with low value bidders or, on the other hand, 0.94 in the auction with high value bidders, the likelihoods of non-participation are, respectively, 0.75, 0.79 and 0.72 , consistent with $2.55,2.08$ and 2.78 active bidders. If the auction is then doubled in size, so that $N=20$, the expected numbers of active bidders become 2.71, 2.31 and 2.89.

Table 1 also hints, however, that both the threshold and expected number of active bidders will be sensitive to the costs of participation. When there are 10 potential bidders whose private values are drawn from the uniform distribution, for example, an increase in costs from 0.01 to 0.05 causes 
the threshold to rise, from 0.63 to 0.74 , and the expected number of active bidders to fall, from 3.69 to 2.59. Curiously, perhaps, almost the same number (1.10) of active bidders are "lost" under other distributions: $1.14=3.69-2.55$ when the distribution is bell-shaped, $1.11=3.19-2.08$ when it is skewed to the left, and $1.16=3.94-2.78$ when it is skewed to the right.

We know, from the previous section, that the participation threshold will be lower in second price auctions, and the results in Table 2 provide some sense of the difference in practice. In the extreme case of $N=2$ potential bidders with low participation costs, there is no threshold at all. That is, both bidders will participate, no matter what their private values. In fact, in auctions with few(er) low value bidders, in particular when the distribution of private values is either $F(v \mid 2,2)$ or $F(v \mid 5,1)$, the threshold is zero even when costs are 0.10 . To understand this, recall that in the case $N=2$ - or, with $N>2$ potential bidders, the sub-case $M=2$ - the representative bidder knows that she will either win the auction or determine what the winner pays and therefore the public benefits that accrue to both bidders. This is sometimes sufficient to induce low value bidders to participate, despite the costs.

While full participation is a special feature of (some) "minimal" or $N=2$ second price auctions, the difference remains substantial as auction size increases. In the uniform case, the increase in the threshold under either the first price or all-pay mechanisms, from 0.10 to 0.79 , for example, as the number of potential bidders increases from 2 to 20 when costs are 0.01 , stands in marked contrast to the increase from 0 to 0.62 under the analogous second price mechanism. In an auction with 20 potential bidders, this is the equivalent of an almost $85 \%$ increase in the number of active bidders, from 4.11 to 7.58. Nor is the size of this effect an artifact of the choice of distribution function: for the same auction size and participation costs, the numbers of expected bidders are 4.06 and 7.56 when the distribution is $F(v \mid 2,2), 3.60$ and 7.18 when it is $F(v \mid 1,3)$, and 4.27 and 7.74 when it is $F(v \mid 5,1)$. In short, in the absence of cost differentials, it seems that second price auctions will be more "active," and to the extent that this is a secondary objective for the charity, a point in their favor.

Otherwise, the same broad patterns characterize participation across mechanisms. The expected number of active bidders, for example, is not all that sensitive to the distribution of private values, but is responsive to variations in cost. Under the bell-shaped distribution, for example, the expected number of active bidders when $N=20(7.56)$ and costs are 0.01 is almost identical to that under the uniform (7.58), and not far from those in the left (7.18) and right-skewed (7.74) distributions, but as costs rise to 0.05 , the expected number of active bidders falls to 6.66 . 


\subsection{Bid Functions}

Consider, for comparison purposes, the familiar result that in a first price auction without spillovers or participation costs, bidders whose values are drawn from a uniform distribution will "shade" their bids by an amount equal to $\left(\frac{1}{N}\right)^{\text {th }}$ of their value, and bid $\frac{N-1}{N} v$. This is depicted, for $N=15$, as the solid line in the upper left panel in Figure 2a, in which various first price bid functions have been plotted. Relative to this benchmark, the introduction of revenue proportional benefits $(\alpha=0.25)$ and warm glow $(\gamma=0.10)$, represented in the same panel by the dotted line, seems to function like an ad valorem subsidy to bidders, an observation easily substantiated on the basis of (8): when $\underline{v}=0$ and $F(v)=v, \sigma^{f}(v)$ is equal, after some simplification, to $\frac{N-1}{(1-\gamma) N-\alpha} v$, or $\frac{\alpha+\gamma N}{(1-\gamma) N-\alpha}$ percent more than was bid in their combined absence.

Under some conditions, the subsidy is sufficient to reverse bid shading. In the diagram, a bidder whose private value is 1 , for example, will bid 1.057; in general, $\sigma^{f}(v)$ will exceed $v$ under the uniform distribution when $\alpha+\gamma N>1$, an inequality that seems likely to be satisfied in most large auctions. Furthermore, the subsidy is increasing in both the common return $\alpha$ and warm glow $\gamma$, as expected, and decreasing in the number of potential bidders $N$.

The further addition of participation costs equal to 0.05 has dramatic effects on the bid function, as the dashed line in the same panel reveals. The behavior of bidders is now sharply nonlinear, for example, and the reason is not just the introduction of a substantial threshold - indeed, to the extent that bids are not zero, but undefined, below the threshold, this is no reason at all - but instead the pronounced concavity of the bid function above the threshold. Close to the threshold, bids increase very rapidly and then level off, a feature with important econometric implications. As a result, the effect of participation costs on the value of the average bid, as opposed to the number of bidders, is quite limited: a bidder who decides to participate knows that if others follow suit, their values must (also) be quite high, and therefore bids aggressively. A bidder whose value is close to the maximum (1), for example, bids almost as much as she would in the absence of participation costs.

The fourth and final function plotted (as a series of dots and dashes) in the same panel is the equilibrium bid function when the common return, warm glow and participation cost remain in place, but the number of potential bidders is reduced to $N=5$. It serves as a reminder that a standard result on auction size and first price bids - that bidders with more competitors are more aggressive because they cannot afford to shade their bids as much - doesn't hold in this environment, at least not for all values. In visual terms, the reason is that the smaller auction also has a lower threshold, so that a bidder who is indifferent about participation when $N=15$, and who would 
therefore submit a zero bid if she did participate, would find it in her interest to submit a positive bid when $N=5$. For high value bidders, the "shading effect" appears to dominate; for low(er), but still above the second threshold, value bidders, the "participation effect" does, another important consideration in the estimation of bid functions.

The other panels in Figure 2a show the same four bid functions for the three alternative value distributions, and suggest that these results are robust. Consider what is perhaps the least similar case, the situation depicted in the lower left panel in which there is a preponderance of low value bidders. It should come as no surprise that even in the standard case - that is, no common return, no warm glow, and no costs of participation - bids are no longer proportional to values: because (small) variations in private value do not have much effect on the likelihood that a high value bidder will win in this environment, bids are not adjusted much either. Furthermore, unlike the uniform case, bidders never bid more than their values, at least for the parameter values considered here.

This said, the two panels share at least three important features. First, it still appears that in the absence of participation costs, the introduction of a common return and warm glow have much the same effect on bids as an ad valorem subsidy. Second, those with values close to the maximum aren't much affected by participation costs or, in broader terms, the effects of these costs on bid behavior diminish with value. Third, with both shading and participation effects at work, high and low value bidders respond quite differently to an increase in auction size.

The characterization of second price bid functions is much less complicated. First and foremost, the four panels in Figure 2b provide visual confirmation that with the common return and warm glow present, variations in the number of potential bidders $N$ or participation costs $c$ influence the participation decision but not, conditional on participation, the bid itself. In effect, there exists a "one size fits all" second price bid function that is "activated" for some combinations of $N$ and $c$ but not others. In the uniform case depicted in the upper left panel, for example, a bidder with private value 0.30 will bid 0.502 when $\alpha=0.25$ and $\gamma=0.10$ when costs $c$ are zero, but not bid (as opposed to a bid of zero) when costs are 0.05 , but another bidder with a value just 0.01 higher will bid 0.511 in both situations.

Furthermore, consistent with intuition, this one size fits all bid function differs across distributions but in all cases reflects some inflation of bids relative to the standard auction, in which it is dominant to bid one's value, no matter what the distribution of values. This inflation no longer resembles an ad valorem subsidy, however, as it did in first price auctions. Under a uniform distribution, for example, the difference declines not just in proportional, but absolute, terms as value increases, from $0.242(=0.242-0.00)$ when $v=0$ to $0.11(=1.11-1.00)$ when $v=1$. The same is true when 
the distribution of values is either hump shaped or skewed to the right, but not when it is skewed left, when the difference increases from 0.094 when $v=0$ to 0.111 when $v=1$. Since the difference between standard and charity-inflated second price bids does not vary much across distributions for high value bidders - indeed, is the same for bidders with $v=1$ - the explanation is found in the differences for low value bidders.

Consider, for example, second price auctions with a preponderance of high value bidders which, as illustrated in the lower right panel of Figure $2 \mathrm{~b}$, produces the largest difference in the behavior of low value bidders: a bidder whose value is close to zero will bid almost nothing, for example, in the absence of common return and warm glow, but more than 0.75 in their presence. The intuition is that in the (expected) presence of many high value bidders, the benefits to low value bidders of an inflated bid - in particular, the possible increase in the "second price" and therefore auction revenues and common return - exceed the costs of an improbable "win."

Casual inspection of the all-pay bid functions in Figure 2c suggests that the effects of participation costs are less pronounced than in either first or second price auctions. To illustrate with the familiar uniform case, consider the behavior of the median potential bidder, someone with $v=0.50$. Since the first price and all-pay thresholds are the same, we know that such a bidder will not participate in the charity auction when there are $N=15$ potential bidders and costs are 0.05. Even when participation is costless, however, the optimal bid is less than one one hundredth of one percent of her value or, to be more precise, $4.38 \times 10^{-5}$, which is itself a substantial (in proportional terms, that is) inflation of the optimal bid in a standard auction, $2.85 \times 10^{-5}$.

For some, if not most, econometric purposes, the difference between a bid, however small, and no bid at all is all that matters, but it is not clear whether someone whose optimal bid is close to 0 will - or, faced with the indivisibilities that constrain bidders in most real world auctions, even could - participate. This in turn has important, if still unexplored, consequences for other bidders.

The uniform case also exhibits the predictable bid inflation associated with charity auctions, one that, in this case, increases in absolute, but decreases in relative, terms. It also demonstrates that the common view that increased competition restrains bidders when bids are forfeit does not hold in the presence of participation costs. (In fact, it doesn't hold in their absence, either: from (36), the optimal bid function when $c$, and therefore $\underline{v}$, are zero, is $\frac{N-1}{N} \frac{1}{1-\beta} v^{N}$, the value of which must (only) eventually decline in $N$.) In this case, the upper left panel of Figure $2 c$ reveals that high value bidders, at least, are more aggressive when $N=15$ than $N=5$. In broader terms, the difference in thresholds causes the bid functions to cross once, a pattern reminiscent of first price auctions: for low(er) values in their common domain, bids are smaller with $N=15$ than $N=5$, while the 
opposite is true for high(er) values.

Unlike the first price auction, however, even the behavior of very high value bidders is sensitive to the existence of participation costs. The so-called "maximal bidder" will bid 1.36 in a charity auction with participation costs of 0.05 , and 1.44 in the same auction without such costs.

All of these features are robust with respect to the distribution of private values, or at least the four distributions considered here.

Finally, Figure 3 allows for the comparison of bid functions across mechanisms and distributions in the special, if now familiar, case of $N=15$ potential bidders, participation costs $c=0.05$, common return $\alpha=0.25$ and warm glow $\gamma=0.10$. The surprise, perhaps, is how little can be said about the relative sizes of bids across mechanisms. One obvious exception is that for all values in their common domain, second price bidders bid strictly more than their first price counterparts, a result that carries over from standard auctions. It is not even the case that both are always more aggressive than those who must forfeit their bids under the all-pay format; in fact, for three of the four distributions pictured here, those with very high values will bid more in all-pay than either first or second price auctions. The intuition for this is that with revenue proportional benefits, such bidders are, in effect, subsidized by their rivals. This is consistent with the observation that the exception is the distribution associated with a preponderance of low value bidders, depicted in the lower left panel: under these conditions, the common return is never sufficient to rationalize bids well in excess of private values.

This said, under all four distributions, all-pay bids are smallest for low(er) value bidders, and remain so over much of the common domain before surpassing (at least) first price bids, a consequence of the fact that all-pay bidders forfeit their bids, no matter what the outcome of the auction.

\subsection{Revenue Functions}

Our principal interest here are not the bid function itself, but their revenue implications. To this end, consider Figure 4a, which plots the variation in expected revenue as a function of auction size $(N)$ across both distributions and mechanisms. Its most obvious feature is that in every case, revenue rises, at a diminishing rate, with the number of potential bidders. Because this is not inevitable - recall that Menezes and Monterio (2000) find that participation costs cause revenues to rise and then fall in the absence of a common return - the result serves to cast doubt on the practical significance of such anomalies.

Furthermore, with the limited exception of the $F(v \mid 1,3)$ distribution, expected revenue more or less levels off after the first dozen or so potential bidders. A similar pattern characterizes the 
standard auction, but the explanation is a little different: in the standard case, the first order statistic for private values is a concave function of the number of bidders with an upper limit of 1 , the upper bound of the distribution of values, but in charity auctions with endogenous participation, this is amplified by the fact that as auction size increases, the number of active bidders also increases at an ever diminishing rate. The map from potential to active bidders also helps to explain the fact that revenues in the low value $F(v \mid 1,3)$ auction do not level off as soon: as a review of Tables 1 and 2 reveals, there are fewer active bidders, ceteris paribus, in this environment.

Some will be surprised that even with $N=40$ potential bidders, the first and second price mechanisms produce such different revenue. The problem is that here, too, intuition is based on the case of compact distributions and costless participation. From (8) and (23), it follows that in both cases, the winner's payment, and therefore auction revenue, are equal to $\sigma^{f}(1)=\sigma^{s}(1)=(1-\gamma)^{-1}$, no matter what the distribution of values.

This leads us to broader conclusions about the relative performance of mechanisms. Figure 4a suggests that at least two inequalities are robust with respect to the distribution of private values: for any number of potential bidders $N$, both the second price and all pay formats "revenue dominate" their first price equivalent. Both inequalities are consistent with previous results for auctions with a fixed number of active bidders (that is, costless participation) and have the same intuition.

The response of the second price/all pay revenue differential to variations in the number of potential bidders is more complicated, but not much so. Under all four distributions, the all pay mechanism eventually produces more revenue, in expectation, than its second price equivalent. For auctions with either a uniform or bell-shaped distribution of values, it happens almost at once - that is, when there are 3 or more potential bidders - and for the auction with a preponderance of high value bidders, it holds even in the limiting case $N=2$. It is only when there is a preponderance of low value bidders that the second price mechanism does better in auctions of intermediate size (under the assumed parameter values, $N$ less than 30). To understand this, recall that with so many low value bidders, high value bidders aren’t subsidized enough to bid very aggressively.

Figure $4 \mathrm{~b}$, which depicts the relationship(s) between expected revenue and participation costs for auctions with $N=10$ potential bidders, leads to some important, if unexpected, conclusions. Consistent with intuition, revenues decline as participation costs rise, across both distributions and auction formats. In the case of second price auctions, however, the decline is almost imperceptible: if private values are uniformly distributed, for example, expected revenue declines from 0.953 when $c=0$ to 0.937 when $c=0.15$, or 30 percent of the median value. From an operational perspective, charities that do not know what it costs to participate in their auctions will sometimes find that 
the second price mechanism serves them best, despite the results in Figure 3a. To understand this, recall that in second price auctions, cost influences the decision to participate but not, conditional on participation, the bid itself.

The fact that the all pay mechanism is (much) more cost sensitive than the second price leads to an important reversal: consistent with intuition, the all pay format is more lucrative for charities when there are no, or even few, obstacles to participation, but as participation becomes more difficult, the premium shrinks and is eventually reversed. Both, however, do better than the first price mechanism no matter what the costs of participation.

\section{Conclusion}

The model described in this paper provides a first framework for the study of charity auctions with endogenous participation. At the cost of a smaller number of hard and fast rules, it can explain what some researchers have observed in the field and introduce some new considerations into the choice of mechanism.

For example, to return to one of the questions that motivated this paper, namely, is the mere existence of participation costs sufficient to explain the underperformance of the all-pay mechanism reported in Carpenter, Holmes and Matthews (2006)? The short answer is no. Consider, for example, the variation in participation rates across mechanisms, and recall that in this field experiment, more bidders were "active" under the first price mechanism than either the second price or all-pay mechanisms, which implies that $\underline{v}^{f}<\underline{v}^{s}<\underline{v}^{a}$. If the obstacles to participation are not mechanismspecific, so that $c^{f}=c^{s}=c^{a}$, the model instead predicts that participation rates in second price auctions will exceed those in either first price or all-pay auctions, or $\underline{v}^{s}<\underline{v}^{f}=\underline{v}^{a}$. Under the maintained assumptions of the model, this requires, at a minimum, that the costs of participation in a first price auction $c^{f}$ be smaller than either alternative. Without additional information, the participation data cannot pin down the relationship between $c^{s}$ and $c^{a}$, however: more bidders will participate in second price auctions even if it costs a little more to do so.

The revenue data support, perhaps even refine, these conclusions. Carpenter, Holmes and Matthews (2006) found that in the field, the first price mechanism produced more revenue than either the second price or all-pay mechanisms, which in turn were more or less "revenue equivalent," or $R^{f}>$ $R^{s} \approx R^{a}$. Further examination of Figure 3b suggests, however, that within the framework of the model, this outcome is not just inconsistent with constant (across mechanism) costs of participation, but implies that $c^{f}$ is less than both $c^{s}$ and $c^{a}$. Once more, the relationship between $c^{s}$ and $c^{a}$ seems 
elusive, but it is also reasonable to infer that the two mechanisms would not produce the same revenue under different circumstances unless $c^{s}<c^{a}$, that is, unless it cost bidders more to participate in an all-pay auction.

We find support for this in some preliminary survey data. In lab experiments conducted by Carpenter, Holmes and Matthews (2007), participants were askeds to assess the fairness and complexity of different mechanisms on a scale from 1 to 5 , where 1 represented least fair and easiest to comprehend, respectively. The results suggest that the all-pay format was perceived as significantly less fair $($ mean $=1.97)$ than the English auction $($ mean $=4.0)$, the silent auction $($ mean $=3.77)$ and the raffle $($ mean $=3.54)$ and significantly more difficult to understand (mean $=2.07)$ than both the English $($ mean $=4.0)$ and silent $($ mean $=1.58)$ auctions and the raffle $($ mean $=1.28)$.

\section{References}

Anderson, Jenny. 2007. Big names, big wallets, big cause. New York Times, May 4, C-1.

Andreoni, James. 1995. Warm-glow versus cold-prickle: the effects of positive and negative framing on cooperation in experiments, Quarterly Journal of Economics, Vol. 110: 1-21.

Carpenter, Jeffrey, Jessica Holmes and Peter Hans Matthews, 2006. Charity auctions: a field experiment, forthcoming, Economic Journal.

Carpenter, Jeffrey, Jessica Holmes and Peter Hans Matthews, 2007. An introduction to "bucket" auctions for charity, Department of Economics, Middlebury College, working paper.

Davis, Douglas, Laura Razzolini, Robert Reilly and Bart J. Wilson, 2006. Raising revenues for charities: auctions versus lotteries, in Douglas Davis and R. Mark Isaac, editors, Research in Experimental Economics, Vol. 11. New York: JAI Press: 49-95.

Engelbrecht-Wiggans, Richard. 1994. Auctions with price-proportional benefits to bidders, Games and Economic Behavior, Vol. 6: 339-346.

Engers, Maxim and Brian McManus. 2006. Charity auctions, International Economic Review, forthcoming.

Goeree, Jacob K., Emiel Maasland, A. Sander Onderstal and John L. Turner. 2005. How (not) to raise money, Journal of Political Economy, Vol. 113: 897-918. 
Goeree, Jacob K. and Arthur Schram. 2003. Bidding to give: an experimental comparison of auctions for charity. CREED Working Paper.

Kumaraswamy, P. 1980. A generalized probability density function for double-bounded random processes, Journal of Hydrology, Vol. 46: 79-88.

Menezes, Flavio M. and Paulo K. Monteiro. 2000. Auctions with endogenous participation, Review of Economic Design, Vol. 5: 71-89.

Samuelson, William F. 1985. Competitive bidding with entry costs, Economics Letters, Vol. 17: $53-57$. 


$$
\text { Participation Cost }=0.01
$$

Threshold Share of Inactive

Value

$(1,1) \quad \mathrm{N}=2$

$\mathrm{N}=5$
$\mathrm{~N}=10$
$\mathrm{~N}=20$

$$
0.10
$$

0.40

0.63

0.79

Bidders

$(2,2)$

$\mathrm{N}=2$

$\mathrm{N}=5$

$\mathrm{N}=10$

$\mathrm{N}=20$

$(1,3)$

$\mathrm{N}=2$
$\mathrm{~N}=5$
$\mathrm{~N}=10$

$\mathrm{N}=10$

$(5,1)$

$N=2$
$N=5$
$N=10$
$N=20$

\subsection{7}

\subsection{6}

$$
0.63
$$

0.74

\subsection{6}

0.19

0.32

0.44

0.46

0.80

0.90

0.95
Participation Cost $=0.05$

$\begin{array}{cc}\text { Threshold } & \text { Share of Inactive } \\ \text { Value } & \text { Bidders }\end{array}$
0.10
0.40
0.63
0.79
0.06
0.38
0.80
0.17
0.48
0.68
0.82
0.02
0.33
0.61
0.79

Value

$\begin{array}{ll}0.22 & 0.22 \\ 0.55 & 0.55 \\ 0.74 & 0.74 \\ 0.86 & 0.86 \\ & \\ 0.30 & 0.17 \\ 0.57 & 0.54 \\ 0.70 & 0.75 \\ 0.79 & 0.86 \\ & \\ 0.14 & 0.36 \\ 0.29 & 0.64 \\ 0.41 & 0.79 \\ 0.51 & 0.88 \\ & \\ 0.61 & 0.08 \\ 0.87 & 0.49 \\ 0.94 & 0.72 \\ 0.97 & 0.86\end{array}$

Participation Cost $=0.10$

$\begin{array}{cc}\text { Threshold } & \text { Share of Inactive } \\ \text { Value } & \text { Bidders }\end{array}$

$\begin{array}{ll}0.32 & 0.32 \\ 0.63 & 0.63 \\ 0.79 & 0.79 \\ 0.89 & 0.89 \\ & \\ 0.38 & 0.26 \\ 0.63 & 0.63 \\ 0.74 & 0.80 \\ 0.82 & 0.90 \\ & \\ 0.20 & 0.49 \\ 0.35 & 0.73 \\ 0.46 & 0.84 \\ 0.56 & 0.91 \\ & \\ 0.68 & 0.15 \\ 0.90 & 0.58 \\ 0.95 & 0.78 \\ 0.98 & 0.89\end{array}$

\section{Table 1. Threshold Values and Non-Participation Rates Under The FP and AP Mechanisms}

This table reports the threshold value and share of bidders who are inactive under either the FP or AP mechanism for various numbers of potential bidders and participation costs under uniform $(1,1)$, hump-shaped $(2,2)$, left-skewed $(1,3)$ and right-skewed $(5,1)$ distributions of private values. 


$$
\text { Participation Cost }=0.01
$$

$\begin{array}{cc}\text { Threshold } & \text { Share of Inactive } \\ \text { Value } & \text { Bidders }\end{array}$

(1,1) $\quad \begin{aligned} & \mathrm{N}=2 \\ & \mathrm{~N}=5\end{aligned}$

$\mathrm{N}=5$
$\mathrm{~N}=10$

$\mathrm{N}=20$

(2,2) $\quad \mathrm{N}=2$

$\mathrm{N}=5$

$\mathrm{N}=10$

$\mathrm{N}=20$

$(1,3)$

$\mathrm{N}=2$
$\mathrm{~N}=5$
$\mathrm{~N}=10$

$\mathrm{N}=10$

$\mathrm{N}=20$

$$
\begin{aligned}
& N=2 \\
& N=5 \\
& N=10 \\
& N=20
\end{aligned}
$$

$$
\begin{aligned}
& 0.00 \\
& 0.21 \\
& 0.44 \\
& 0.62
\end{aligned}
$$$$
\text { Bidders }
$$

\subsection{0}

$$
0.32
$$$$
0.62
$$

\subsection{0}

0.10

0.19

0.29

0.00

0.70

0.84

0.91
Participation Cost $=0.05$

$\begin{array}{cc}\text { Threshold } & \text { Share of Inactive } \\ \text { Value } & \text { Bidders }\end{array}$

Value

0.00
0.30
0.51
0.67

0.00
0.40
0.55
0.65

0.03
0.14
0.23
0.32

0.00
0.76
0.87
0.92

Participation Cost $=0.10$

$\begin{array}{cc}\text { Threshold } & \text { Share of Inactive } \\ \text { Value } & \text { Bidders }\end{array}$

$\begin{array}{ll}0.04 & 0.04 \\ 0.35 & 0.35 \\ 0.54 & 0.54 \\ 0.69 & 0.69 \\ & \\ 0.01 & 0.00 \\ 0.44 & 0.34 \\ 0.57 & 0.54 \\ 0.66 & 0.69 \\ & \\ 0.06 & 0.18 \\ 0.17 & 0.42 \\ 0.25 & 0.57 \\ 0.33 & 0.70 \\ & \\ 0.00 & 0.00 \\ 0.79 & 0.30 \\ 0.88 & 0.52 \\ 0.93 & 0.68\end{array}$

Table 2. Threshold Values and Non-Participation Rates Under The SP Mechanism

This table reports the threshold value and share of bidders who are inactive under either the SP mechanism $(\mathrm{a}=0.25, \mathrm{~b}=0.35)$ for various numbers of potential bidders and participation costs under uniform $(1,1)$, hump-shaped $(2,2)$, left-skewed $(1,3)$ and right-skewed $(5,1)$ distributions of private values. 


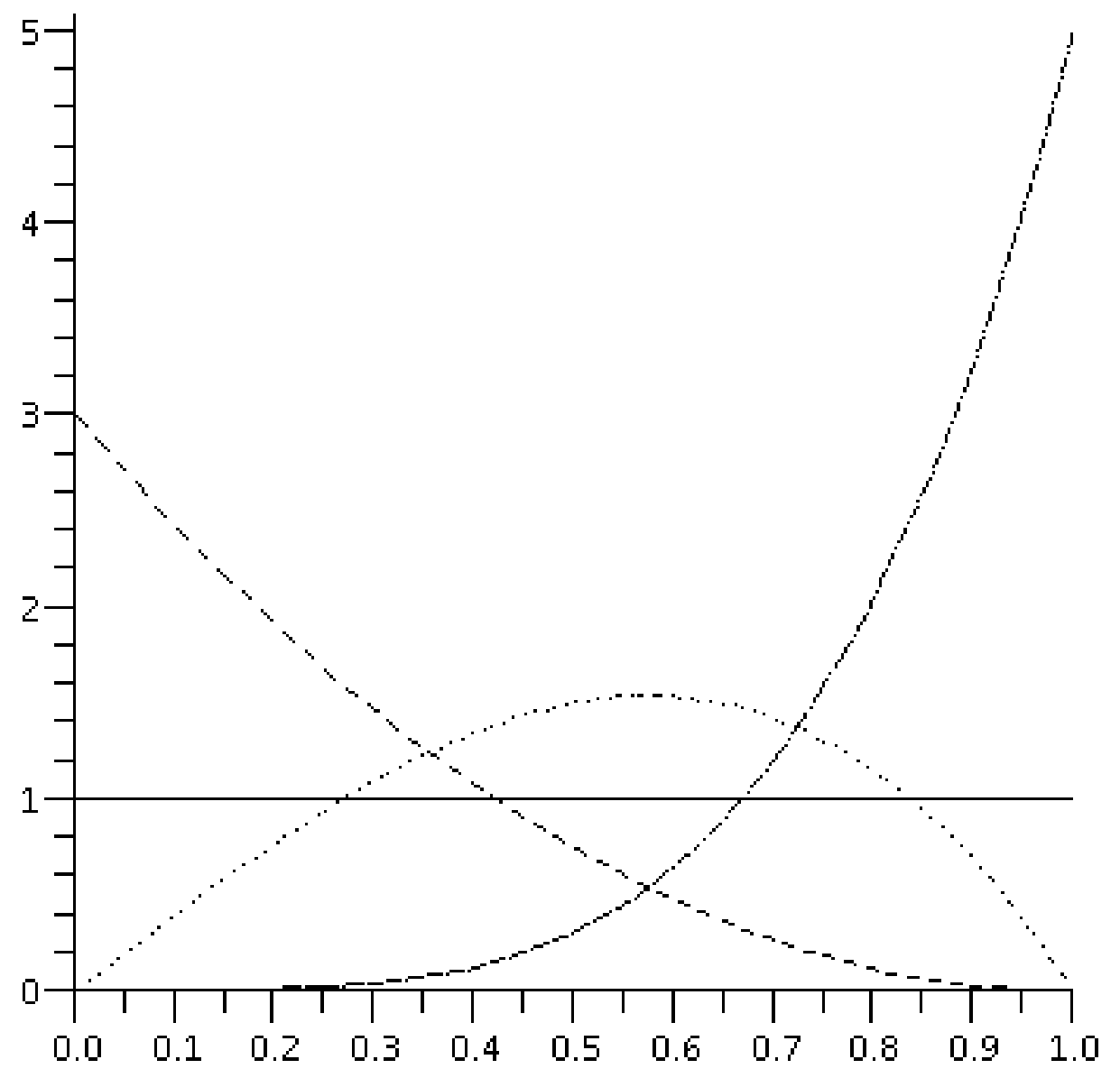

Figure 1. Kumuraswamy Density Functions

Legend: Solid: $a=1, b=1$. Dotted: $a=2, b=2$. Dashed: $a=1, b=3$ Dotted/Dashed: $a=5, b=1$ 
First Price, (1,1) Distribution

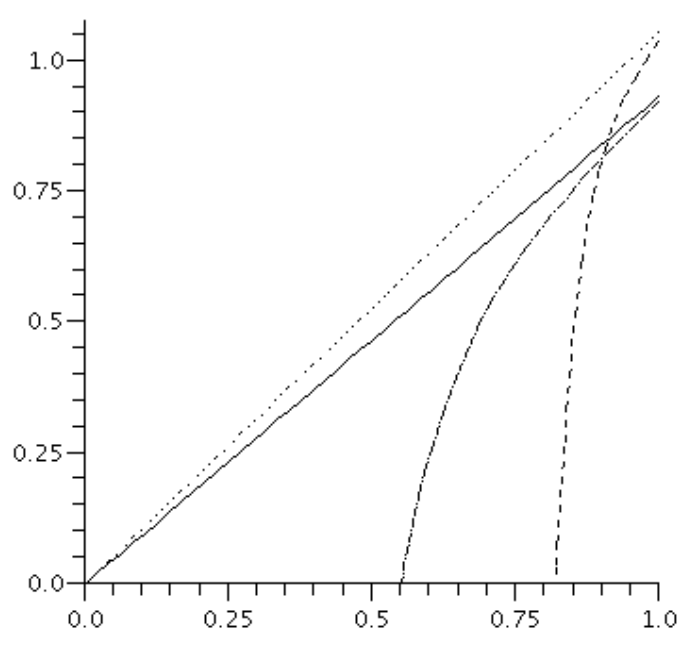

First Price, (1,3) Distribution

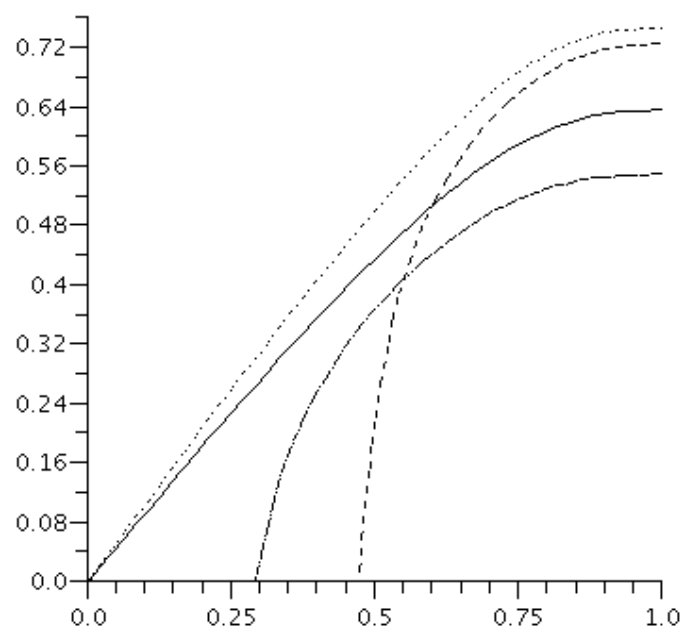

First Price, (2,2) Distribution

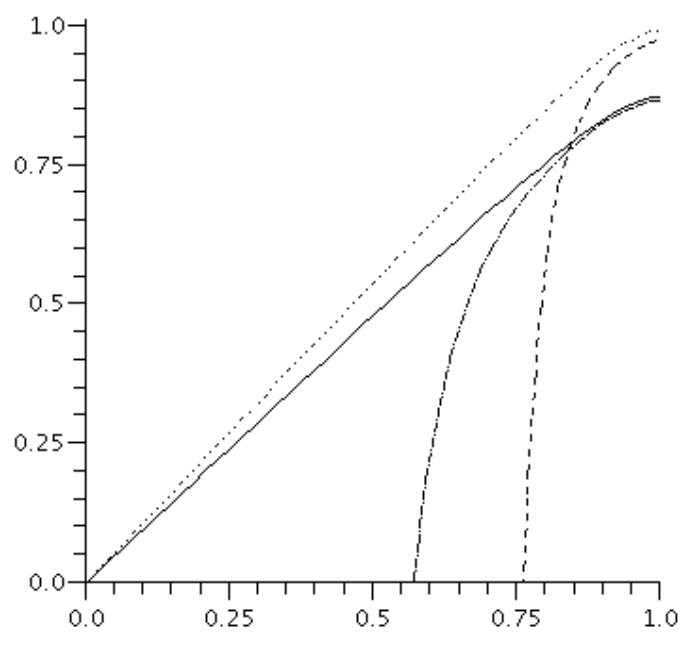

First Price, (5,1) Distribution

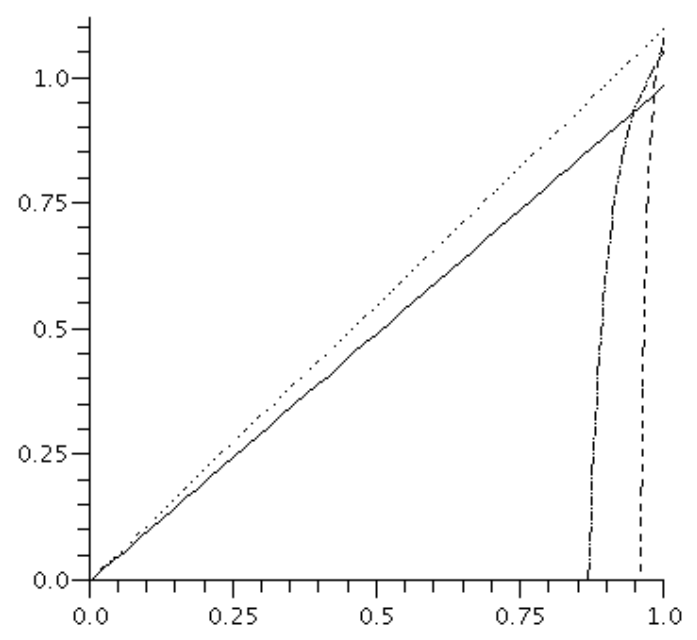

Figure 2a. Optimal Bids in FP Auctions As A Function of Private Value Under Uniform $(1,1)$, Hump-Shaped $(2,2)$, Left-Skewed $(1,3)$ and Right-Skewed $(5,1)$ Distributions.

Legend: Solid:

Dotted:

Dashed:

Dotted/Dashed:

$$
\begin{aligned}
& \alpha=0, \beta=0, C=0, N=15 \\
& \alpha=0.25, \beta=0.35, C=0, N=15 \\
& \alpha=0.25, \beta=0.35, C=0.05, N=15 \\
& \alpha=0.25, \beta=0.35, C=0.05, N=5
\end{aligned}
$$


Second Price, (1,1) Distribution

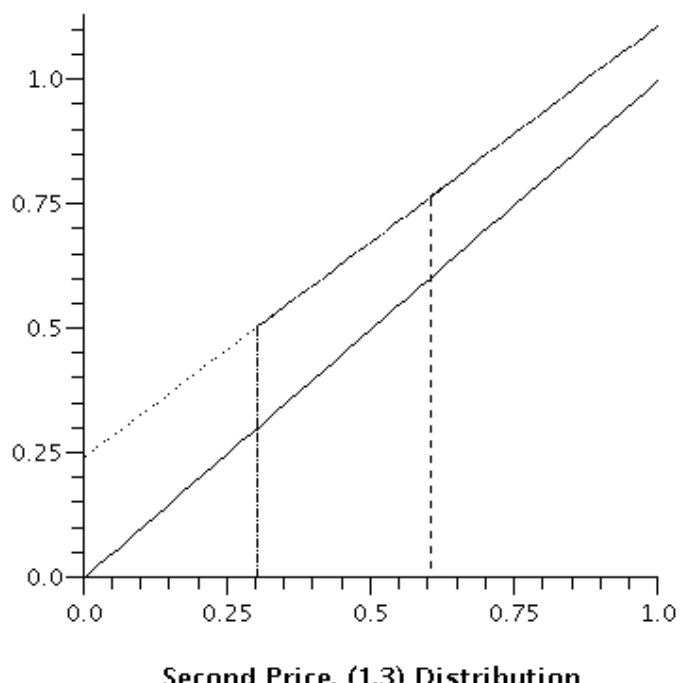

Second Price, (1,3) Distribution

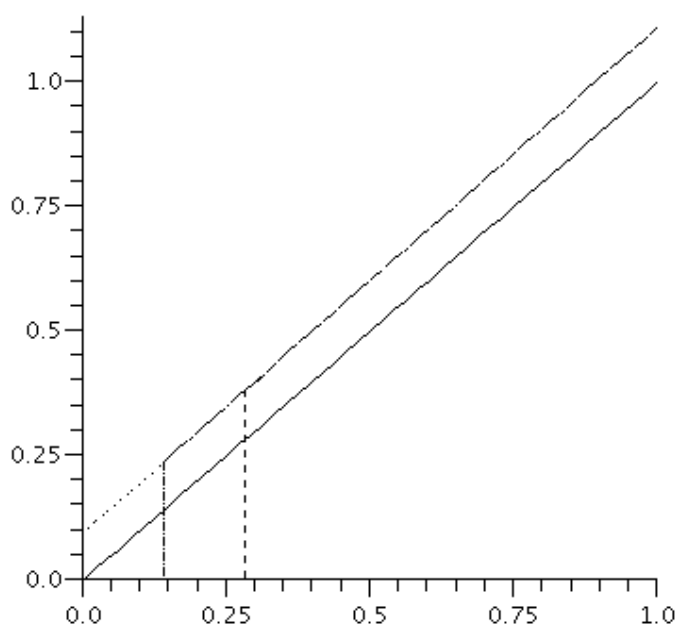

Second Price, (2,2) Distribution

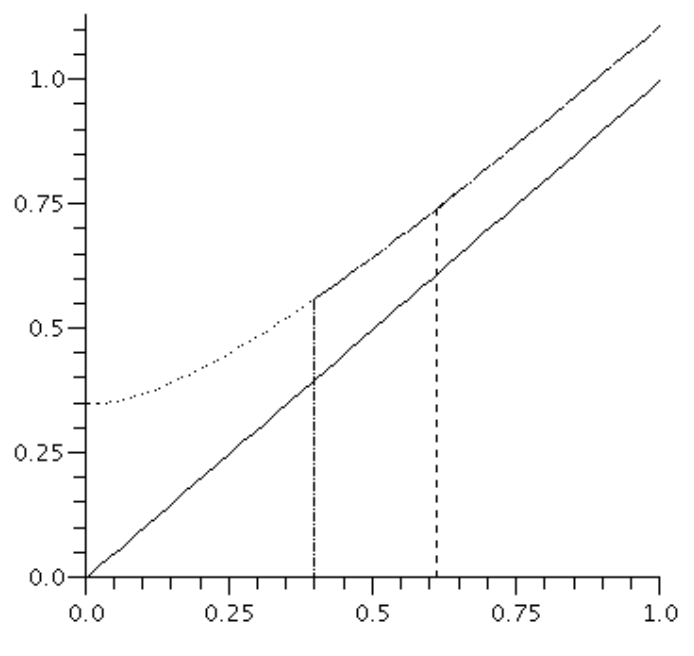

Second Price, (5,1) Distribution

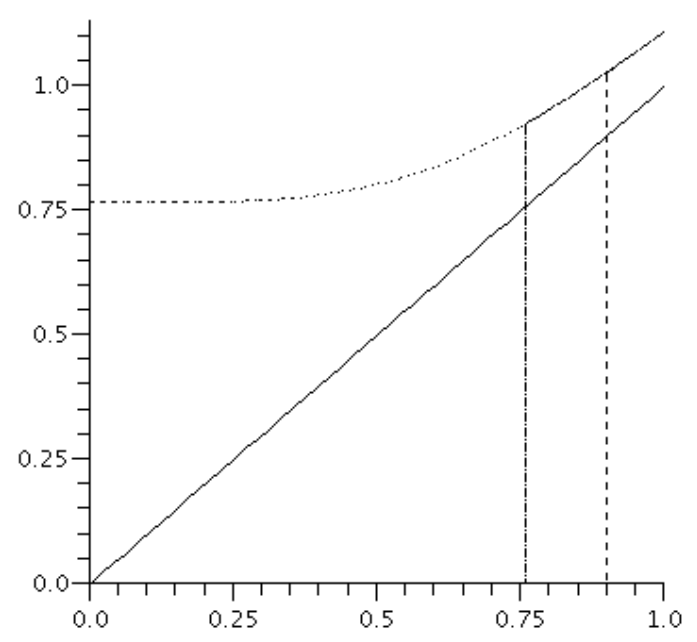

Figure 2b. Optimal Bids in SP Auctions As A Function of Private Value Under Uniform $(1,1)$, Hump-Shaped $(2,2)$, Left-Skewed $(1,3)$ and Right-Skewed $(5,1)$ Distributions.

Legend: Solid:

Dotted:

Dashed:

Dotted/Dashed:

$$
\begin{aligned}
& \alpha=0, \beta=0, c=0, N=15 \\
& \alpha=0.25, \beta=0.35, c=0, N=15 \\
& \alpha=0.25, \beta=0.35, c=0.05, N=15 \\
& \alpha=0.25, \beta=0.35, c=0.05, N=5
\end{aligned}
$$


All Pay, (1,1) Distribution



All Pay, (1,3) Distribution

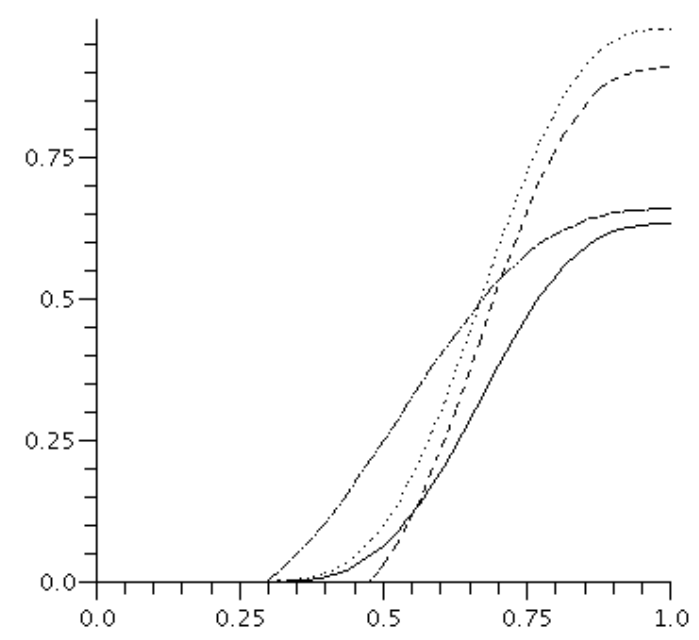

All Pay, (2,2) Distribution

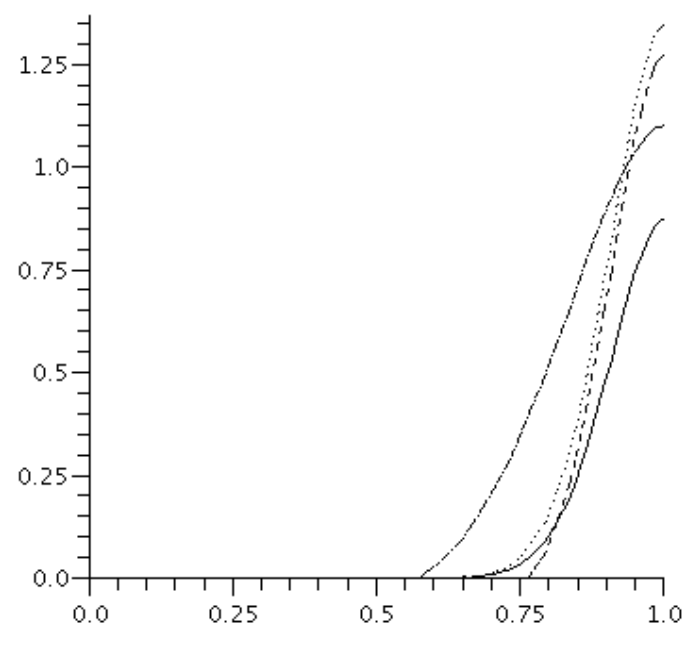

All Pay, (5,1) Distribution

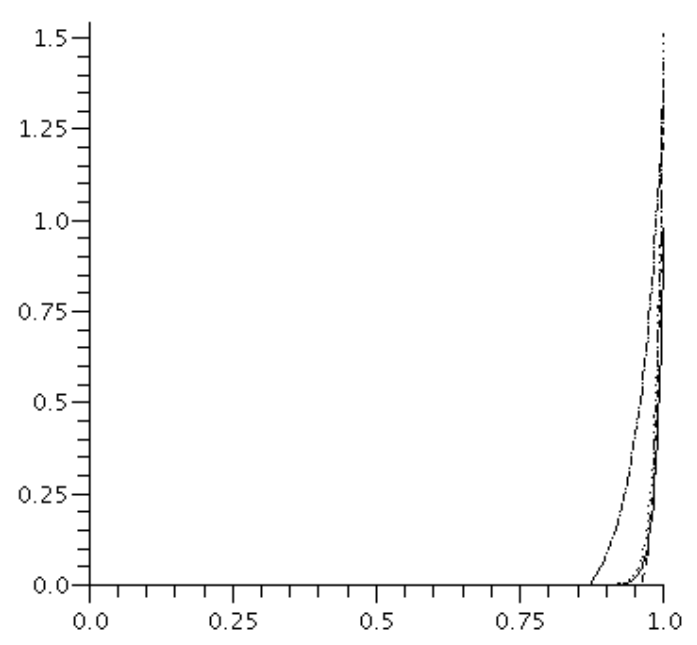

Figure 2c. Optimal Bids in AP Auctions As A Function of Private Value Under Uniform $(1,1)$, Hump-Shaped $(2,2)$, Left-Skewed $(1,3)$ and Right-Skewed $(5,1)$ Distributions.

Legend: Solid:

Dotted:

Dashed:

Dotted/Dashed:

$$
\begin{aligned}
& \alpha=0, \beta=0, c=0, N=15 \\
& \alpha=0.25, \beta=0.35, c=0, N=15 \\
& \alpha=0.25, \beta=0.35, c=0.05, N=15 \\
& \alpha=0.25, \beta=0.35, c=0.05, N=5
\end{aligned}
$$


FP, SP and AP Bids Under $(1,1)$

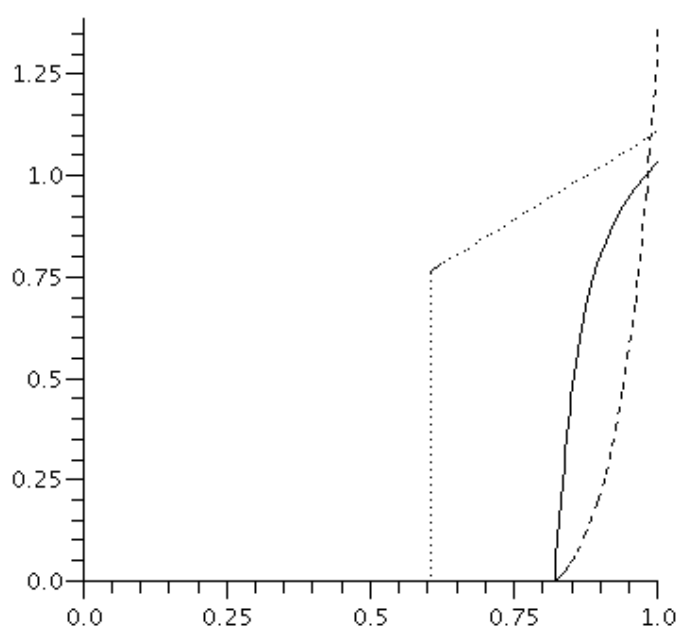

FP, SP and AP Bids Under (1,3)

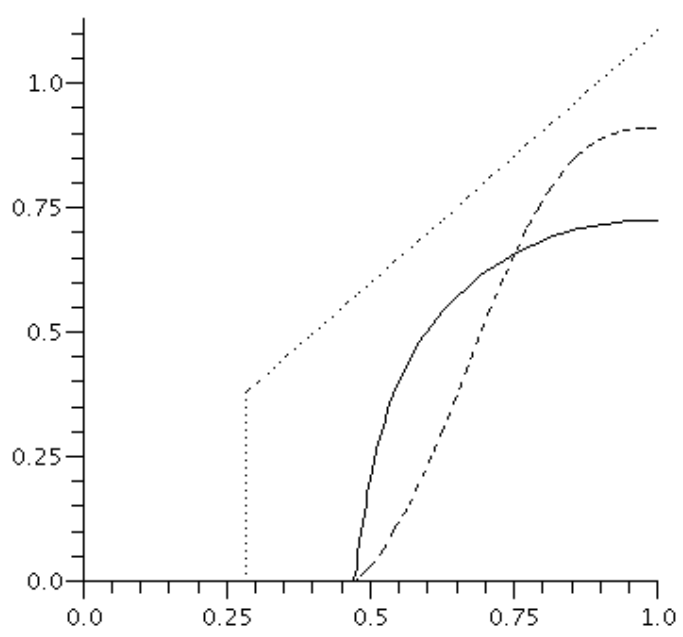

FP, SP and AP Bids Under $(2,2)$

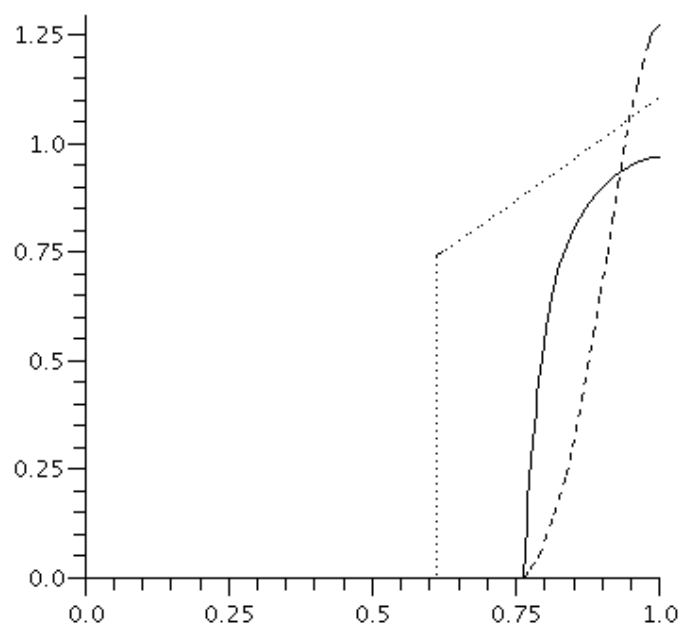

FP, SP and AP Bids Under $(5,1)$

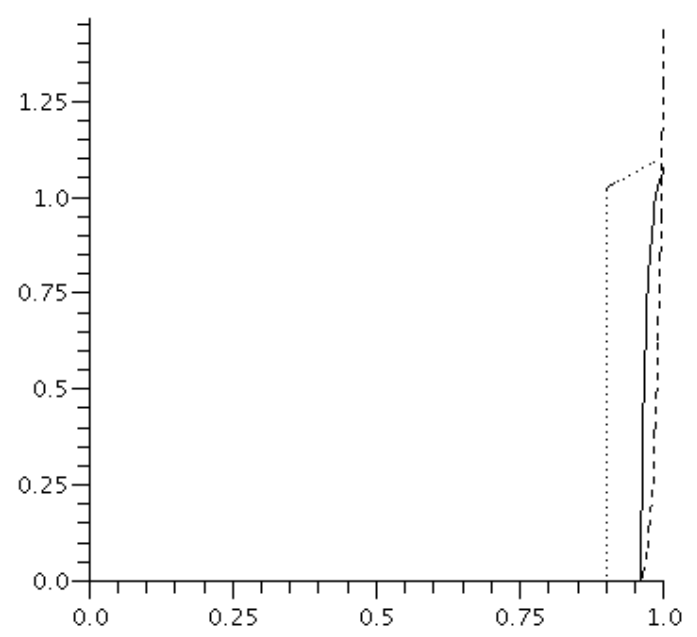

Figure 3. Optimal Bids in FP, SP and AP Auctions Under Uniform $(1,1)$, Hump-Shaped $(2,2)$, Left-Skewed $(1,3)$ and Right-Skewed $(5,1)$ Distributions.

Legend: $\quad$ Solid: $\quad F P, \alpha=0.25, \beta=0.35, c=0.05, N=15$ Dotted: $\quad$ SP, $\alpha=0.25, \beta=0.35, c=0.05, N=15$ Dashed: $\quad$ AP, $\alpha=0.25, \beta=0.35, c=0.05, N=15$ 
The $(1,1)$ Case

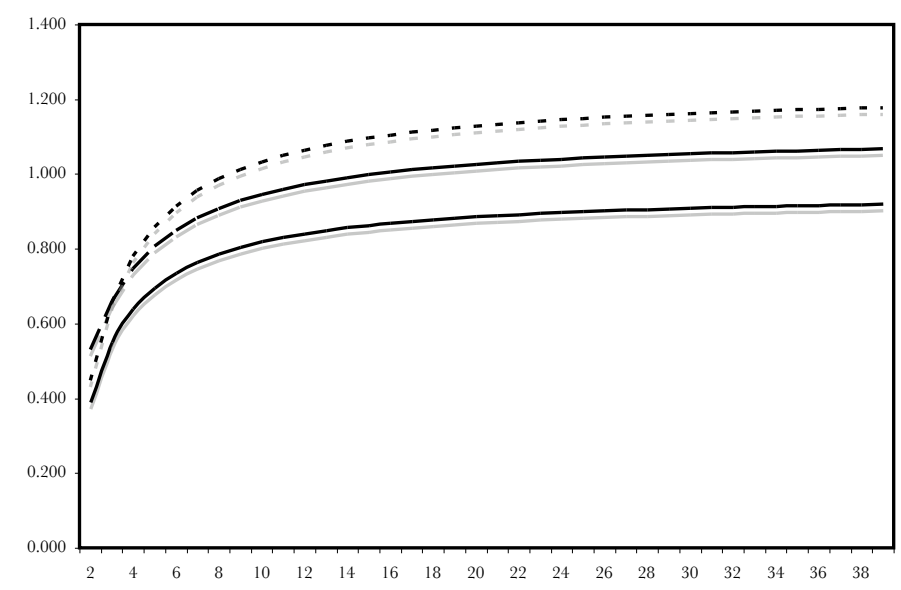

The $(1,3)$ Case

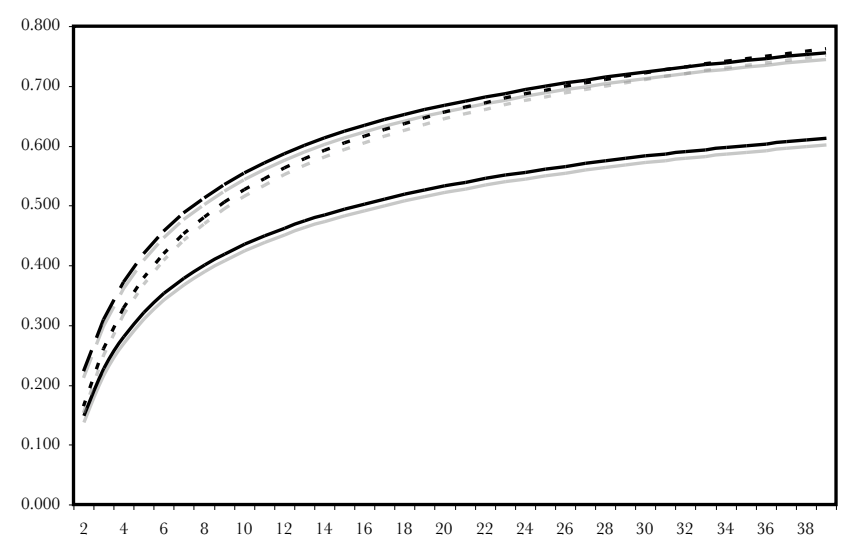

The $(2,2)$ Case

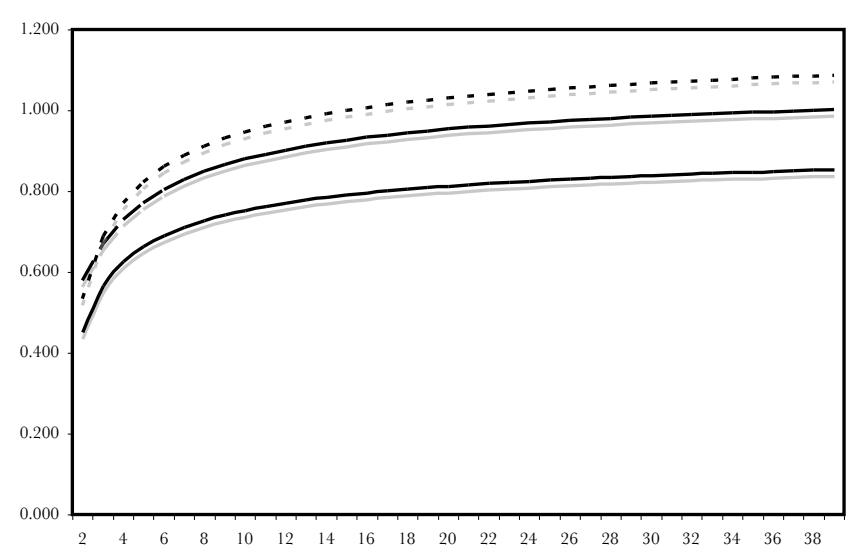

The $(5,1)$ Gase

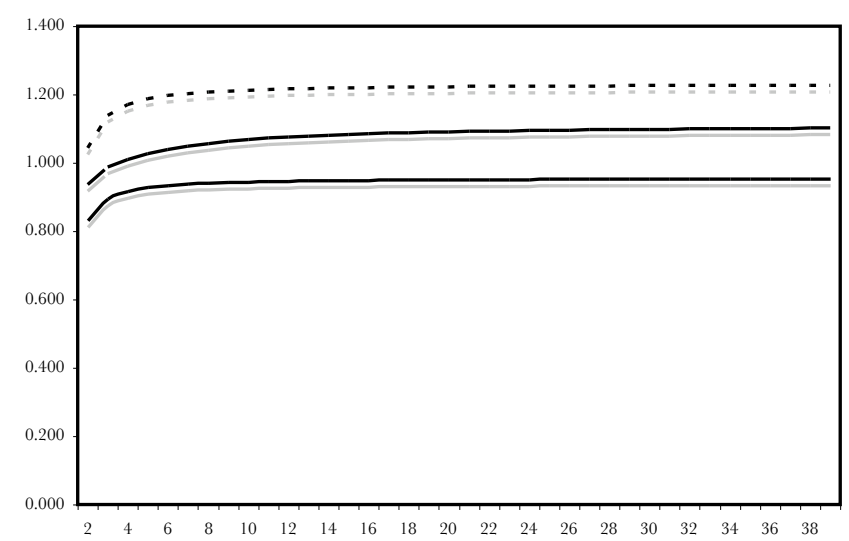

Figure 4a. Expected Revenue as a Function of the Number of Potential Bidders, $\alpha=0.25, \beta=0.35$ and $\mathrm{c}=0.05$ Legend: Solid Line - FP

Dashed Line - SP

Dotted Line - AP 
The $(1,1)$ Case

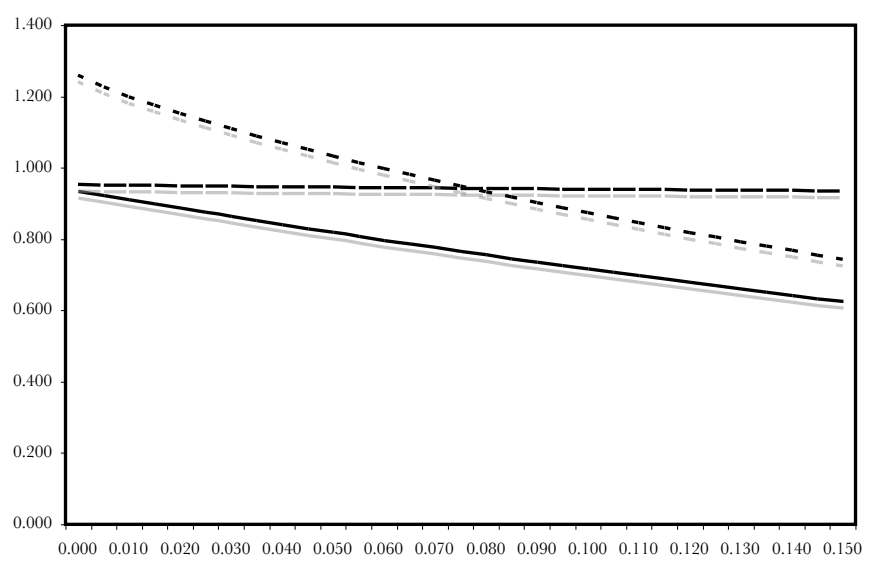

The $(1,3)$ Case

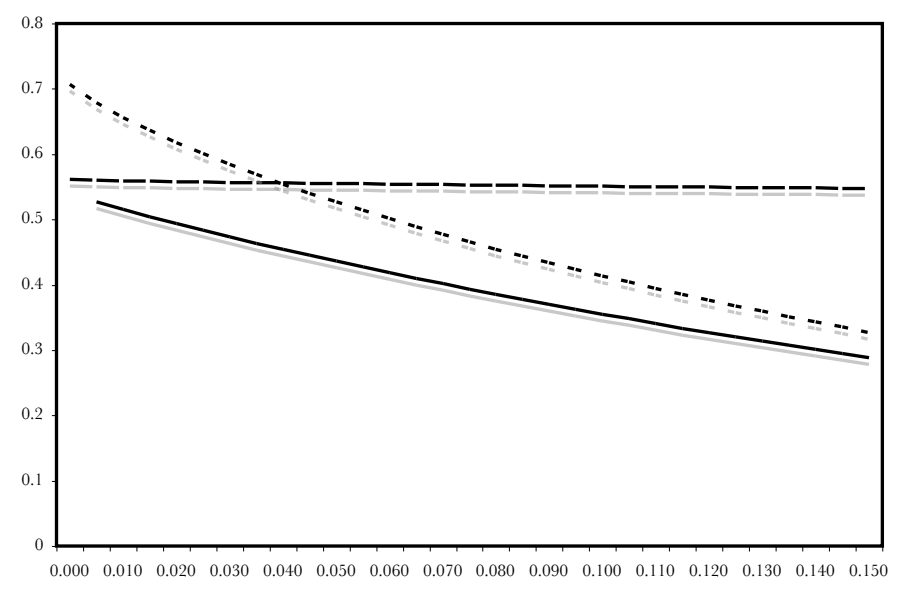

The $(2,2)$ Case

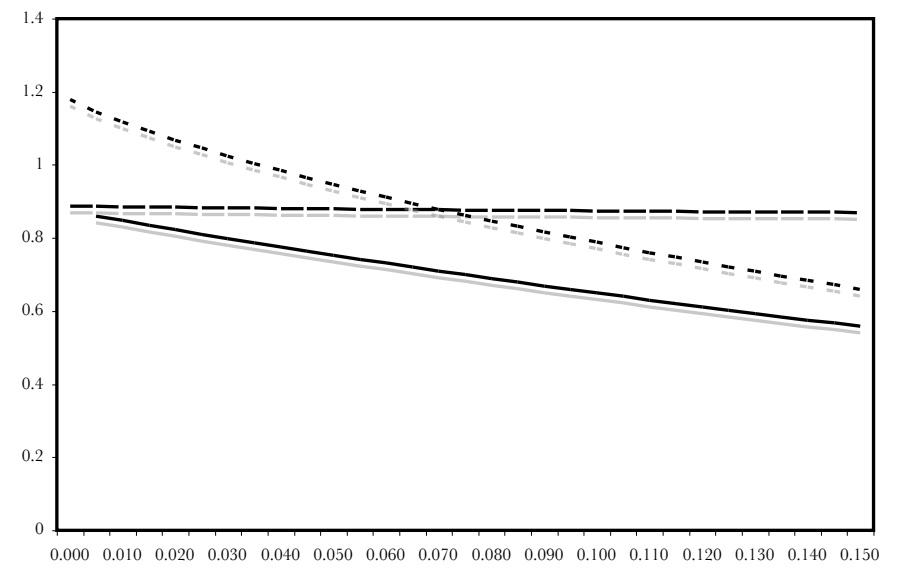

The $(5,1)$ Case

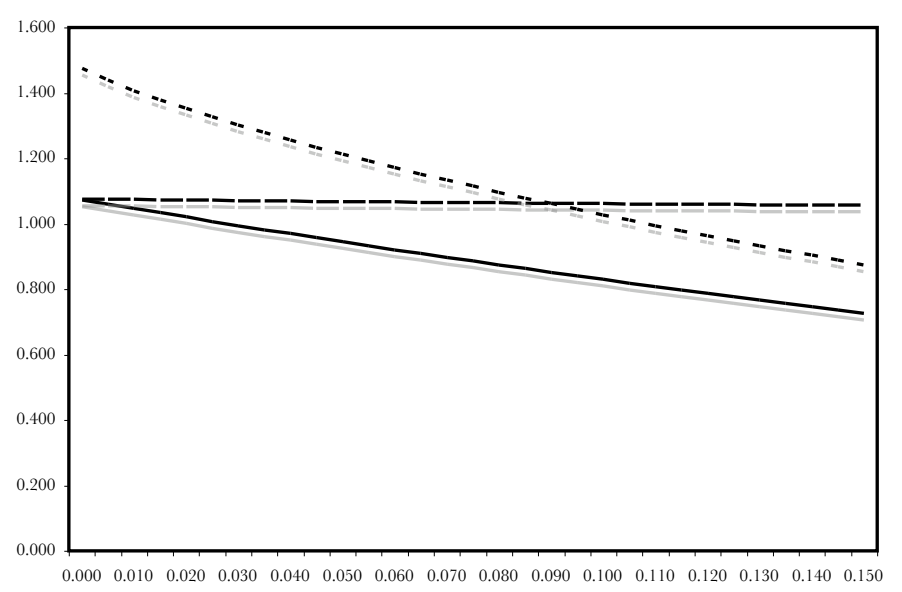

Figure 4b. Expected Revenue as a Function of Participation Cost, $\alpha=0.25, \beta=0.35$ and $N=10$ Legend: Solid Line - FP Dashed Line - SP

Dotted Line - AP 